\title{
Using the brain's fight-or-flight response to predict, prevent and recover from mental illness during NASA's deep space missions
}

\author{
Len Losik \\ President \& Chief Technology Officer, Failure Analysis, Capitola, California, USA
}

Email address:

lenlosik@failureanalysisco.com

\section{To cite this article:}

Len Losik. Using the Brain's Fight-or-Flight Response to Predict, Prevent and Recover from Mental Illness during NASA's Deep Space Missions. American Journal of Applied Psychology, Vol. 2, No. 4, 2013, pp. 31-51. doi: 10.11648/j.ajap.20130204.11

\begin{abstract}
The cause(s) and cure(s) of mental illness eludes the medical health industry allowing today's pharmaceutical industry to drive the treatment for all people with mental illness using drugs that minimize the symptoms allowing people to return to the lifestyle that exacerbated it. It is more profitable for pharmaceutical companies to develop drugs to treat the symptoms than cure or prevent mental illnesses. Deep space missions provide a harsh environment for astronauts, one that may increase the rate and severity of mental illness. Mental illness has been reported by astronauts in low earth orbit and some astronauts have become severely mentally after resuming other responsibilities. Looking at mental illness using electrical engineering, communications science and predictive science, the effects on the brain can be better quantified and understood in defining the cause, prevention and the recovery from mental illness if it is acquired during a deep space mission. This paper proposes that a person's fight-or-flight response be used to explain the cause, prevention and recovery from mental illness due to no other available model in the mental health community. By accepting the FOFR as the cause of mental illness, it can also be used to explain the cause of most other diseases through its control of a person's immune system when activated. Each time a person's immune system is suppressed by the FOFR, it increases the likelihood of contracting a viral illness including cancer and many other diseases the immune system paralyzes. The results of this paper can be applied for general use by encouraging pharmaceutical companies to develop the drugs necessary to suppress the FOFR and subsequently stop the onset of most viral diseases, cancer and mental illness; much as drugs are used to suppress a person's immune system to treat autoimmune diseases.
\end{abstract}

Keywords: Space Medicine, Predictive Diagnostics, Predictive Algorithms, Data Driven Algorithms, Model-Based Algorithms, Mental Illness, Predicting Mental Illness, Preventing Mental Illness, Recovering from Mental Illness

\section{Introduction}

Most definitions for mental illnesses in the public domain describe mental illnesses as a brain disorder followed by a list of symptoms. Many mental health professional believe that this definition defines a mental illness causing great confusing. We will redefine what mental illness is and what it is caused from so that it can be predicted, prevented and recovered from which is not accomplished today.

To help people understand the cause of the symptoms from mental illnesses, a conceptual model allows defining why and how mental illnesses occur. A model also allows the prognostics and health management (PHM) technology to be employed for predicting, preventing and recovering from mental illness while astronauts are on deep space exploration missions.

In science, mathematics and engineering, theories and models are proposed to explain the source of unknown behavior and scientists, mathematicians and engineers have the opportunity to identify the weaknesses and strengths of the theories. If the theories stand up to the rigors of this process, it is adopted until proved wrong and another one is proposed.

This paper will illustrate that using the brain's fight-orflight response (FOFR) as a model to explain the cause, prevention and cure for mental illnesses is valid. It can be confirmed using communication science, predictive science and physiological, biological and engineering 
measurements supplied in later sections taken from an astronauts circadian and ultradian rhythms collected before launch, during launch and while traveling on deep space missions.

To be successful using the FOFR as the model that explains the cause, prevention and recovery from mental illness, we treat mental illness as a communications difficulty between the subconscious and conscious and then apply the communications sciences theories from electrical engineering, to solve for the root cause of mental illness. The mental health community defines mental illness as an unexplainable brain disorder, which requires psychiatric medication for life for treatment.

${ }^{[1]}$ The field in electrical engineering called communications sciences includes the mathematics defining the characteristics for the transfer of information (intelligence) using electrical and/or RF signals including the data bandwidth (speed) in hertz $(\mathrm{Hz})$ required and the quality of the data measured in the bit error rate (BER). To quantify the quality of the information transmitted electrically, all digital bits are counted and the bit-error-rate (BER) is the number of the incorrect digital bits in a fixed number of known bits (often in 1,000,000 bits) transported over some form of communications equipment. Communications science defines the technical characteristics of the transfer of information is done electrically by adding intelligence onto electrical or RF signals.

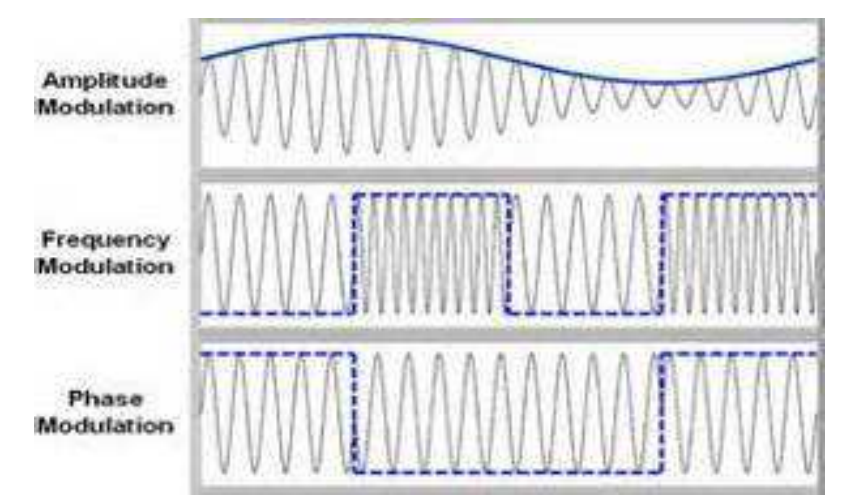

Figure 1. Three Common Modulation Schemes used to add Intelligence onto Electrical and RF Signals using both Analog Sinusoidal Signals and Digital Data Derived from the Analog Data in Communications Sciences

For digital data that is modulated onto a carrier, the higher energy used to create the each digital bit (a.k.a. Eb) compared to the energy in the noise (a.k.a. No) on the carrier, the fewer bit errors occur in the data and the more accurate the message. These data quality items are known and identified, measured and controlled closely in all information communications systems and networks that transfer information in either analog or digital methods.

${ }^{\text {[2] }}$ The communications between cells is done by the tiny transport vesicle package that ship neurotransmitterchemicals to the end (terminals) of the cell and then across synapses, or gaps in-between neurons. Adjacent neurons then receive the signal. To do this, these transport-vesicles must be recycled quickly during boosts in brain activity, but it is not understood how such recycling is done.

${ }^{[3]}$ Including psychiatrists, many professional disciplines that work and research in the field of mental disorders approach mental illness from each of their own viewpoints without a model, speculating on their own conceptual model to explain what they find before them in patients. To understand an individual's mental illness, it is often necessary to borrow from several of these. The on-going debate about the merits of drug treatment versus talk therapy can be viewed as a clash of models: biological versus cognitive.

From Communication Sciences, a field in Electrical Engineering used to design electrical and RF communications systems, analog (continuous) data such as voltage or current, we know that the higher the ratio of the electrical signal levels to the electrical noise level, or S/No, the better the data quality. The lower the S/No ratio, the more likely noise is present that will reduce the quality or accuracy of the message.

Observing proteins within cellular vesicles labeled with a fluorescent marker in the lab, for easy identification, the researchers saw that about 20 vesicles can be simultaneously manufactured right at the end of the neuron that are lined up and waiting to be filled for use. Calcium ions, which help to send the signal across the synapse to another neuron, also control the cell's ability to rebuild the vesicles at the cell's terminal end.

Treating the brain as a closed system allows using communications theory to explain the usefulness of the symptoms, we call mental illness, as a means of communications between the conscious and subconscious. How the symptoms of mental illness are used by the subconscious to communicate with the conscious is explained later.

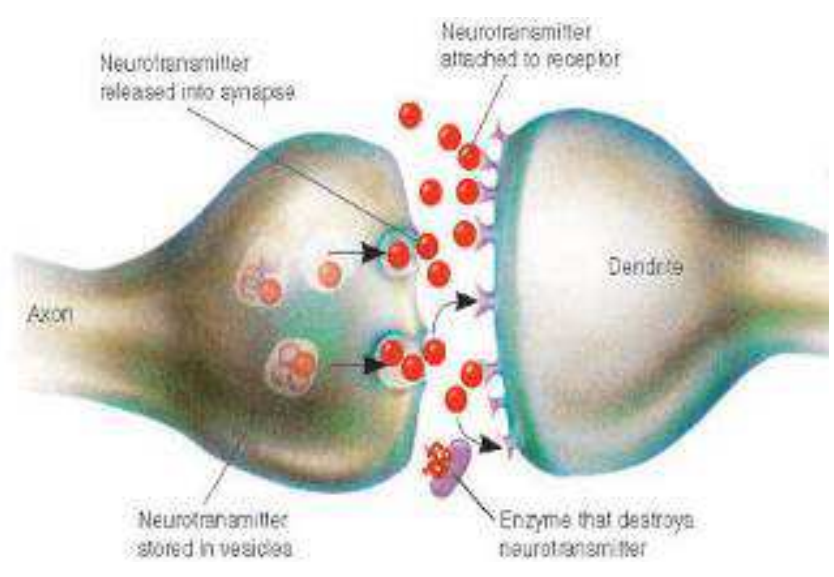

Figure 2. The Synaptic Theory for the Communications Used by the Brain Neurons. ${ }^{[2]}$

The "primitive" portion of the brain receives all the information from all body senses but not receive any information from the conscious, the primitive brain processes all the data from the senses looking for danger and sends a very small portion to the area of the brain that 
creates the conscious mind for the conscious mind to create reality. This one-way communications between the subconscious and conscious is called simplex communications.

Since the FOFR can control any function of the body for any length of time it decides is necessary for selfpreservation, the FOFR may be responsible for most diseases and illnesses including mental illnesses. When the FOFR identifies a threat is present, to ensure an individual's safety, it may activate the FOFR at a high, medium or low level. Whenever the FOFR is activated, it can deactivate the body's immune system allowing all or jus some of the many viruses kept under control to briefly flourish causing a person to get a flu or cold. Since the FOFR was developed for short duration use of activation (milliseconds) when it is activated long term, it can suppress or over excite (autoimmune diseases) the immune system long term and so is likely responsible for most diseases that the immune system normally stops such as arthritis, cancer and other viral related illnesses such as flues and colds. The FOFR can cause the immune system to be over stimulated if it decides it is necessary causing autoimmune diseases such as arthritis.

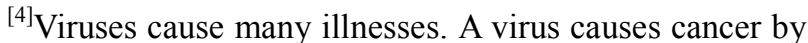
invading a cell and changing the cell's DNA turning on the growth gene. A virus is DNA or RNA wrapped in a protein coat. They do not contain the necessary materials to function on their own so virus's invade a host cell (can be plant, animal, or bacterial) in order to thrive and reproduce.

[3] Advancements in understanding the root cause of premature aging of electrical and mechanical equipment include the development of predictive science and the prognostic analysis included in a prognostic and health management plan (PHM). These are new tools to track the health of spacecraft equipment and changes to an astronaut's brain to identify when the astronaut's brain begins to experience premature aging due to the release of cortisol and adrenaline released when a person's fight-orflight system is activated.

[4] The biological, physiological and engineering diagnostic data necessary to predict, prevent and cure mental illness during deep space missions can be collected from the astronaut's pre-launch spaceflight readiness activities, launch and during actual space flight. Ground personnel can perform a prognostic analysis to identify the presence of accelerated/premature aging in the spacecraft equipment and in the astronaut's brain.

The diagnostic measurements used to predict mental illness originate deep in the brain near the area that generates Circadian and Ultradian rhythms that controls a person's Circadian and Ultranian rhythms used commonly to diagnose many medical conditions. As the brain (and body) age prematurely from the long-term exposure to cortisol and adrenaline, the premature aging can be illustrated using proprietary, dynamic predictive algorithms in a prognostic analysis.

Figure 3 illustrates the new, Congressionally mandated
NASA Space Launch System (SLS) planned to be the next manned launch vehicle that will take astronauts to low earth orbit and to deep space.

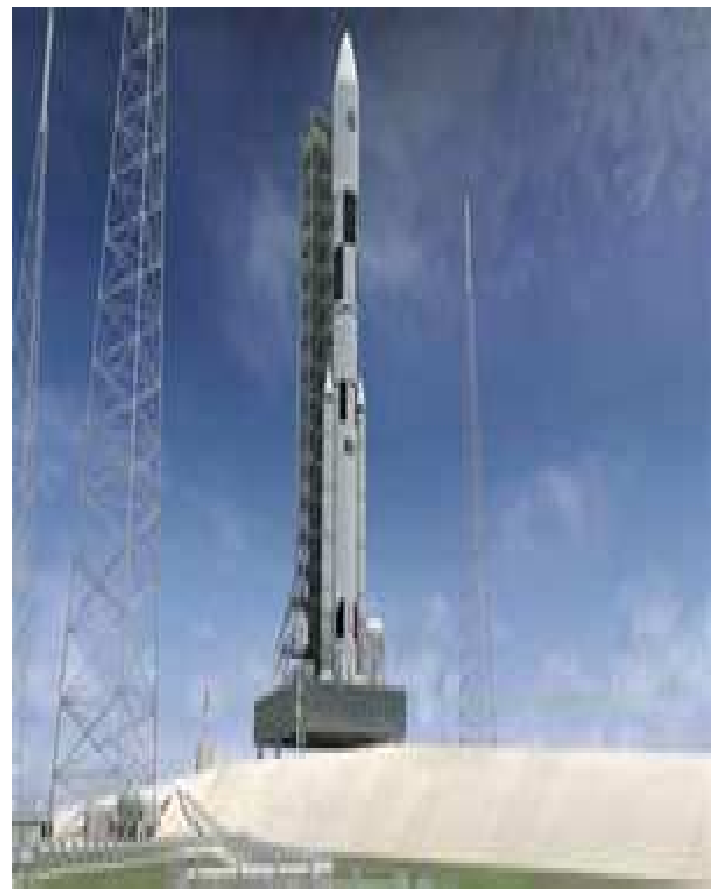

Figure 3. The New NASA Space Launch System is the Congressionally Mandated Replacement to the NASA Manned Space Shuttle that will be used to Launch all Future Manned Space Missions to Deep Space

[5] A PHM program includes using proprietary, dynamic, model-based or data-driven predictive algorithms. These are used to process the biological, physiological and engineering telemetry collected during preflight readiness, prelaunch, launch and on-orbit activities in a scientific analysis. The data used to predict mental illness originates from the astronauts Circadian and Ultradian rhythms that are located deep within a person's brain near where we believe the FOFR originates. A sample of the number and type of measurements to be collected on astronauts are identified in a later section.

${ }^{[6]}$ The area of the brain that the Circadian and Ultradian rhythms originate are next to the area of the brain believed to originate the fight-or-flight response (FOFR) that includes the thalamus, hypothalamus and brain stem that release both cortisol and adrenaline, chemicals toxic to the body with long term exposure causing premature aging.

[7] Anxiety and depression have been reported by astronauts in space during low earth orbiting space missions. The NASA astronaut, Captain Liza Nowak who had extended stays in space exhibited bizarre psychological behavior after she found out that an old boyfriend was dating her friend, demonstrating that the behavior of astronauts with extended stays in space may exhibit more severe psychological behavior than those people who do not train, work and live in space.

${ }^{[7]}$ NASA acknowledges that the human body degrades quickly in $0 \mathrm{~g}$ (e.g. microgravity) in many known and 
unknown ways plus the highly destructive solar radiation environment present outside the earth's atmosphere. ${ }^{[8]}$ An astronaut that has been in space for 6 months loses $9 \%$ to $10 \%$ of its body mass to atrophy without extensive exercise. The effect on the brain cells are unknown but must be considered a serious factor as all other areas of the body cannot perform as they did under $1 \mathrm{~g}$. It is likely that an astronaut's brain atrophies while in space from dying brain cells and scientific studies need to be completed to understand the changes to those people whose body and brains have been exposed to harsh and life-threatening environment of space for extended periods.

Figure 4 is NASA's Astronaut, Captain Liza Nowak. After arrest for attempted homicide, she was diagnosed with major depression, obsessive-compulsive disorder, insomnia, and a psychotic disorder with marked stressors at the time of her attempted homicide of a fellow female astronaut in 2007. Captain Nowak did not use her mental illnesses in her defense, which is common for people with mental illness.

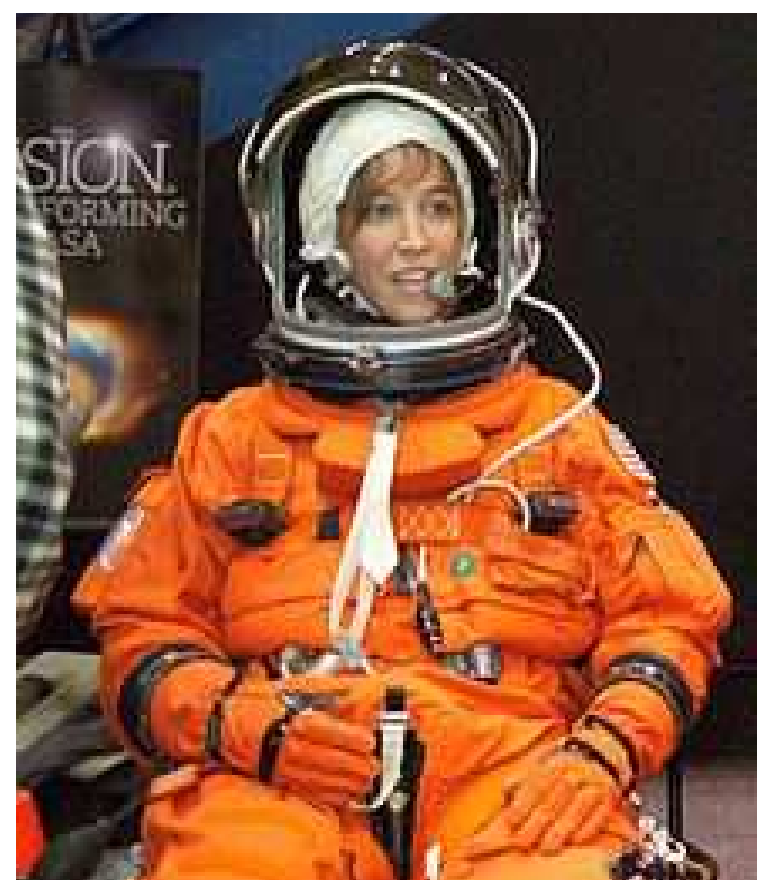

Figure 4. NASA Astronaut Captain Liza Nowak that Suffered from Three Major Mental Illnesses after her Job Reassignment at NASA's Johnson Space Center

[2] The same prognostic tools and techniques used by personnel to predict, prevent and cure mental illness for space applications are also suitable for use by those who are not astronauts and already suffer from mental illness or will suffer from mental illness sometime in the near future. ${ }^{[8]}$ According to the Journal of American Medical Association, $50 \%$ of today's population will acquire a mental illness during their lifetime requiring medication.

\section{Mental Illness, Nature vs. Nurture}

\subsection{Can Mentally Ill Parents Create the Loving and Nurturing Environment Necessary for Children to Develop into a Well Adjusted Person}

First, most mentally ill people are usually not aware that they are mentally ill and do not seek treatment. The mentally ill are often employed, married and have children. Whatever psychological and/or physical trauma the mentally ill parents suffered that caused their mental illness, is often taken as their model and repeated by when they raise their own children to some extent causing their children to also be traumatized. Most all parents will state that they "did the best they could" but sometimes the best we can do is not good enough to raise happy and emotionally healthy children. Some people who have suffered childhood psychological trauma overcome their mental health issues, but parents that are mentally ill while raising their children are far more likely to raise mentally ill children.

\subsection{Why is Mental Illness Predictable?}

${ }^{\text {[2] }}$ For mental illness to be predictable, mental illness must originate solely from a single cause. This single cause is environmental conditions. Some individuals suggest that mental illness is genetically transferred to family members. This belief is based on emotional or mentally ill parents producing children with mental illness and distant family members acquiring mental illness. It is more likely that emotionally and/or mental ill parents will unknowingly reproduce the similar threatening environmental conditions for their children that caused them to acquire emotional or mental illness than those who are raised in a loving and nurturing environment.

Generally, mentally and/or emotionally ill parents cannot be a good judge of whether their home life is a loving and nurturing environment since the environment they were raised was not. It would be unlikely that mentally ill people could create the loving and nurturing environment needed to raise a happy and mentally healthy child.

What may be genetically passed from parent to child are the different thresholds that must be met for mental illness to be acquired. The conscious is one of several interfaces with the world for the subconscious, all information from the senses flows directly to the subconscious first. The information from all the senses is filtered and reduced in amount and sent to the conscious for the conscious to create an individual's "realty." The conscious was developed to increase the likelihood of an animal's survival.

\section{The Manned Space Program}

\subsection{A Harsh and Dangerous Environment}

${ }^{[9]}$ The manned space program can contribute to developing fundamental understanding of the human brain leading to the cause and cures for mental illnesses that have eluded the medical community until know due to the 
extreme conditions that astronauts are exposed to in preparation for getting launched to space and working in space.

${ }^{[9]}$ When working in space, any single movement could mean certain death. The threats while in space include prolonged exposure to solar and galactic radiation, prolonged exposure to microgravity and confinement in close, relatively austere quarters

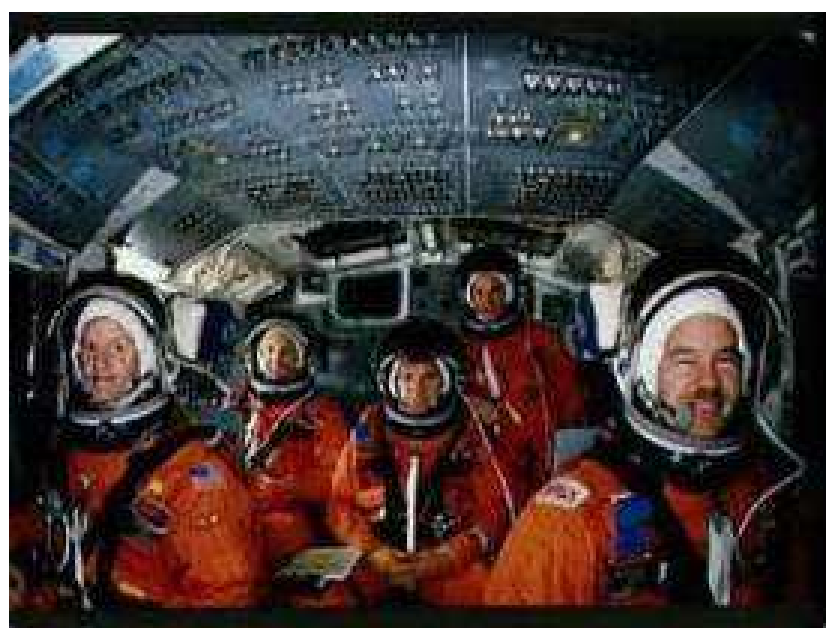

Figure 5. Astronauts Confined in the NASA Space Shuttle Cockpit for Long Duration Deep Space Missions Traveling at 17,000 mph.

\section{Predictive Science}

Predictive science is a new discipline for predicting when a system or a component will exceed it performance or functional requirements with certainty allowing catastrophic failures to be eliminated. Predictive science was developed to replace probability analysis, which uses stochastic (fictional) equations to quantify equipment or product usable life in unreal terms. A lack of functionality and/or performance exceeding specifications is most often a failure and the equipment can no longer be used. The predicted time of failure is referred to as the remaining useful life (RUL) and is the result of a prednostic analysis, which is often important information necessary for risk mitigation.

Predictive science is based on the analysis of failure modes developed using failure behavior from many like units. The illustration of premature aging a.k.a. the early signs of wear and aging, and previously identified fault conditions. These previously identified failure precursors become a damage propagation model and model-based predictive algorithms were first developed to leverage these failure models. Data-driven predictive algorithms were developed after recognizing that non-repeatable transient events (NRTE) were related to equipment end of life and could be used to predict failures with certainty and determine remaining usable life.

\subsection{Predictive Algorithms}

${ }^{[10]}$ A predictive algorithm is a series of actions that may include completing a scientific analysis, made by personnel trained to identify the presence of premature aging that is related to equipment end-of-life. Using predictive algorithms requires performance information from inside the item of interest.

A scientific analysis is much different from an engineering analysis, which allows using speculation and conjecture in the results. Therefore, the results from a scientific analysis are more meaningful for a scientific analysis than an engineering analysis.

${ }^{[11]}$ Using predictive algorithms as part of a prognostic and health management (PHM) program in the manufacture of electrical and mechanical equipment and products will prevent surprise equipment failures from occurring as well as surprise mental illness from occurring. Some technical decisions made in the design phase of complex systems will be different when a PHM is employed in a preventive paradigm compared to the decisions made in a reactive paradigm after a surprise failure occurs.

${ }^{[12]}$ Table 1 is a list of proprietary, dynamic, data-driven predictive algorithms that were pioneered on the Air Force's Global Positioning System (GPS) satellites by the author. These algorithms were used to illustrate the presence of premature aging in the equipment analog telemetry on board each GPS satellite. Prior to processing with predictive algorithms, the telemetry behavior appeared normal and the equipment was fully functional. These algorithms were subsequently used on NASA satellites during dynamic environmental factory acceptance testing, in low earth orbit and a large commercial communications satellite in geosynchronous orbit.

Table 1. A List of Proprietary, Data-Driven Predictive Algorithms ${ }^{[12]}$

\begin{tabular}{ll}
\hline Algorithm Name & Purpose of Algorithm \\
\hline $\begin{array}{l}\text { Baseline } \\
\text { Analysis }\end{array}$ & Identifies short and long term normal data behavior \\
$\begin{array}{l}\text { Change } \\
\text { Analysis }\end{array}$ & Determines change from normal behavior. \\
Comparison Analysis & Determines when a change in normal behavior is occurring \\
Day of Failure & Search large data sets for common behavior during the same time \\
\hline
\end{tabular}




\begin{tabular}{|c|c|}
\hline Algorithm Name & Purpose of Algorithm \\
\hline $\begin{array}{l}{ }^{1} \text { Digital } \\
\text { Processing }\end{array}$ & Replaces outliers improving image accuracy and resolution \\
\hline Discrimination Analysis & Identify behavior that has changed from normal baseline behavior \\
\hline${ }^{2}$ Mathematical Modeling & Generates baseline behavior when little or no data is available \\
\hline Multi-Variant Limit Analysis & Simultaneous analysis across several measurements \\
\hline Rate Change Analysis & Identifies change in behavior from baseline based on rate of change \\
\hline Remaining Usable Life & Determines remaining usable life \\
\hline $\begin{array}{l}{ }^{3} \text { Statistical } \\
\text { Sampling }\end{array}$ & Reduces amount of data without eliminating desired behavior \\
\hline State Change Analysis & Identifies data to be evaluated based on state change \\
\hline Super Impositioning & Identifies data to be analyzed further for failure signature \\
\hline $\begin{array}{l}{ }^{1} \text { Super } \\
\text { Precision }\end{array}$ & Improves data integrity \\
\hline $\begin{array}{l}* \text { Telemetry } \\
\text { Authentication }\end{array}$ & Improves data integrity \\
\hline $\begin{array}{l}{ }^{2} \text { Virtual } \\
\text { Telemetry }\end{array}$ & Creates normal data behavior when none is available \\
\hline $\begin{array}{l}\text { Data } \\
\text { Integration }\end{array}$ & Creates demodulated image for analysis \\
\hline $\begin{array}{l}\text { Dataset } \\
\text { Generation }\end{array}$ & Creates a database manually for completing a prognostic analysis with incompatible data interfaces \\
\hline
\end{tabular}

${ }^{1}$ Algorithms not used when telemetry is provided using CCSDS format. ${ }^{2}$ Algorithm used when little or no diagnostic data is available to create a baseline.

${ }^{3}$ Algorithm used to reduce the amount of data to complete a prognostic analysis.

Most aerospace and defense industry electrical and mechanical equipment provides analog (continuous) telemetry for processing with predictive algorithms. To predict mental illness, information from deep within the brain is needed. It is not feasible to insert wires into the brains of astronauts, but it is possible to determine the performance of certain areas of the brain by measuring the behavior of the body that is controlled by the area of the brain of interest. The areas of the brain believed responsible for the symptoms of mental illness are near the areas of the brain that generate the body's natural rhythms and so measurements from this area of the brain will reflect if premature aging is occurring.

\section{Circadian and Ultradian Rhythms}

${ }^{[6]}$ Physiological rhythms are changes occurring over time with a reproducible waveform. The period of our body's rhythms may range from a fraction of a second, to a few hours, to about 24 hours (circadian rhythms) and even longer. The Earth's daily light-dark cycle governs rhythmic changes in people's behavior and/or physiology. These changes are governed by a biological clock located in two areas of the brain called the suprachiasmatic nuclei.

When the brain experiences premature aging from the FOFR, which is also near the location the body's rhythms are created, it causes the circadian and/or ultradian rhythm to suffer from a variety of mental and physical disorders. These have proven to affect people's safety, performance and productivity. Adverse effects from disrupted circadian and ultradian rhythms are linked to most disturbances in the sleep-wake cycle and vice-a versa. Some rhythmic processes are more affected by the circadian clock than by the sleep-wake state, whereas other rhythms are more dependent on the sleep-wake state.

\subsection{NASA's CCSDS Packetized Telemetry Format}

The quality of the data from spacecraft in deep space is susceptible to many sources of noise that decreases the reliability of the data. The more systems that are used to relay the telemetry, the more bit errors are likely.

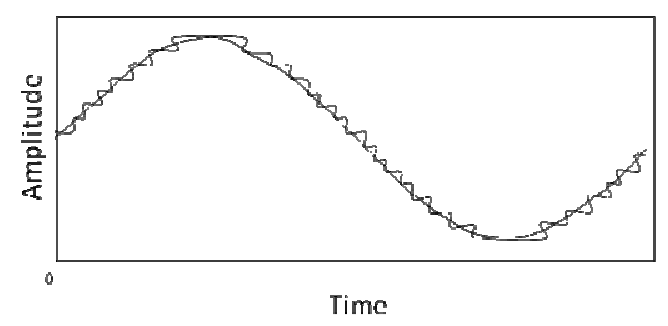

Figure 6. A Comparison between Telemetry and the Original Analog Signal Illustrating that Telemetry is Segmented and Reconstructed Analog Data. 
NASA developed its own packetized telemetry formatting many decades ago that provides high quality telemetry under all known operating conditions. The biological, physiological and engineering diagnostic data needed to predict a person's future medical status is transmitted from space to the ground using a telemetry system that relays telemetry through the NASA TDRSS geostationary communications satellites.

The telemetry interface with equipment provides the engineering performance information outside the equipment for routing to remote locations for processing around the world in real-time. Telemetry measures equipment performance and status and does not a measure equipment remaining usable life. The digitized telemetry generated in space is transmitted to ground facilities and reconstructed into an analog signal using $5^{\text {th }}$ and $6^{\text {th }}$ order polynomials whose sections of the polynomials closely mimics the behavior from the original continuous analog signal.

${ }^{[13]}$ The NASA CCSDS telemetry packet format, generation and routing system is extremely complex and a costly system that ensures high quality communications between space and ground personnel. High quality telemetry is required for completing a scientific analysis because non-repeatable transients occur in telemetry from the communication systems that mimics premature aging and so noise can be a source of false positives or false negatives if the electrical noise in the telemetry is not removed.

\section{What is Mental Illness}

\subsection{Communications between the FOFR and the Conscious}

The symptoms the mental health community defines as mental illness are simply the changes the FOFR makes to a person's behavior to communicate with the conscious to educate the conscious it is making too many dangerous decisions and to start making different, "safe" decisions as determined solely by the FOFR. In its communications with the conscious, the FOFR uses such items as feelings, emotions and hunches. The information input to the conscious mind from the subconscious mind is significantly less than the sensory input into the FOFR subsystem. We call this simplex communications in communications science. The conscious mind would be easily overloaded if it received all the information that the FOFR received from a person's eyes, ears, nervous system, skin and nose, so the subconscious sends a minor amount of information to the conscious for the conscious to create a person's "realty." This implies that each person's "reality" is different from anyone else's reality.

The communications capability between the conscious and subconscious mind is close to zero and this is the reason that the external sensory inputs such as ears, eyes and nose, to the subconscious mind must be used to communicate with the subconscious mind by the conscious mind even though both reside in the same brain.

It is confusing to understand that the conscious thoughts are not accessible to the subconscious mind, but they are not. Data exists that the communications between the subconscious and conscious mind is one way thus forcing the conscious mind to use the same communication channels that are used by the subconscious for receiving all other information.

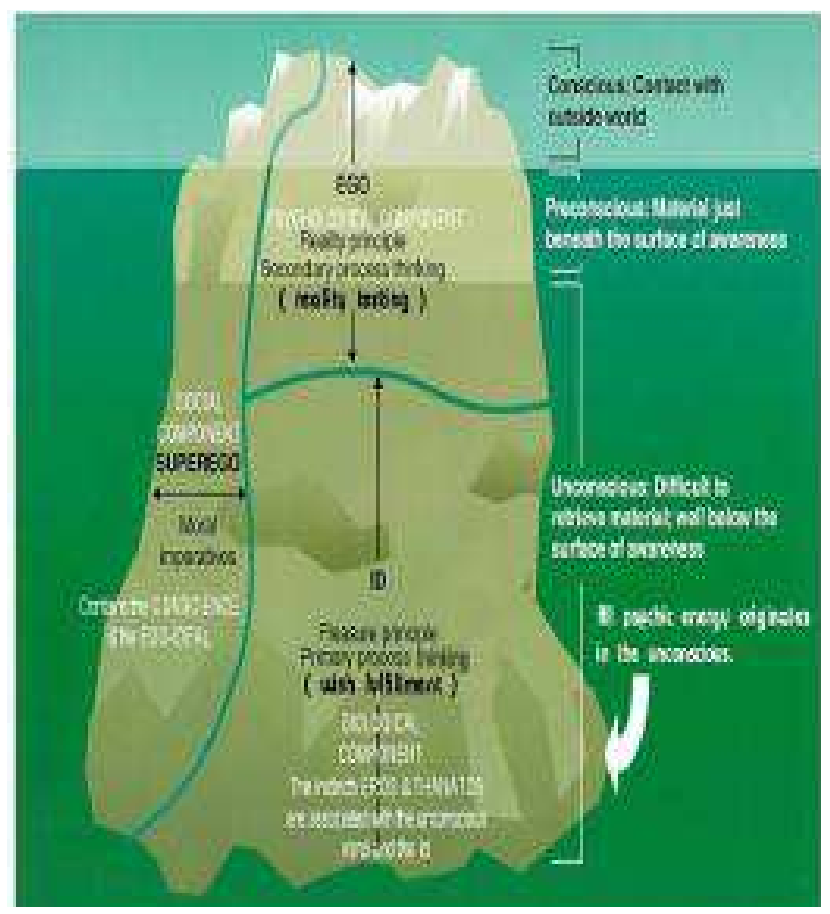

Figure 7. The Resources Allocated between the Conscious and Subconscious Functions of the Complete Mind (Freud).

\subsection{The Recipe for Mental Illness}

Acquiring mental illness can be simplified into several steps, these steps are"

1. Generally from two to five years of exposure to psychological and/or physical danger or trauma as defined by each person's FOFR, resulting in the need for the person to develop psychological "survival" behavior(s). This continuous exposure to danger triggers the near continuous release of cortisol and adrenaline causing premature aging of the body and brain and shrinkage of the brain, resulting in:

a. The FOFR experiencing an overdose of feeling a state of "powerless" to get the conscious to remove the threat

b. The FOFR experiencing a state of being " out-ofcontrol" from the failure of the conscious to remove the threat the FOFR is aware of

c. The development of psychological "survival strategies" including learning to ignore or suppress feelings and emotions that are the messages from the FOFR to the conscious, destroying or degrading the communications capability between the subconscious to the conscious

2. A person is unable to get away from or have the 
conscious remove the threat or hostility,

a. The FOFR then forces the conscious to experience the discomfort from depression. If the conscious continues to make the wrong decisions (as determined solely by the FOFR) resulting in the FOFR deciding that a threat or hostility is still present, the FOFR increases the number and severity of the symptoms the conscious must experience. These symptoms are included as major depression (that is recoverable from) that adds the feelings of despair, hopelessness, helplessness, anxiety and thoughts of suicide.

b. If the decision(s) the FOFR wants the conscious to make are not made, the FOFR initiates then nonrecoverable changes in brain's chemistry that cause more psychological and physical painful symptoms known as bipolar disorder or schizophrenia. These are so severe that psychiatric drugs are required for the person to function. If the FOFR causes schizophrenia, it has began to remove the conscious as its interface to the world, requiring medication to keep the conscious functional

\subsection{When does Mental Illness Occur}

Mental illness occurs when the conscious continuously ignores the messages from the FOFR to either get away from or remove itself from a threat or a series of threats or dangerous conditions the FOFR decides exists. We often call this trauma and the trauma can occur as children and adults.

Mental illness will eventually occur when the conscious continuously fails get away from or remove the threat(s) or, continues returning to a threatening environment for a long period such as to hostile parents or place of employment. If the threat(s) continue to be defined by the FOFR, the subconscious then experiences a state of "powerlessness" and determines the conscious is "out of control" and will not get the conscious to remove the threat of get away from the threat as the FOFR (not the conscious) defines it. These events occur most often to adults who, as children were raised in a long term hostile and/or long term threatening environment (to the FOFR) often including major neglect by their parent(s) and physical or emotional abuse. As a result of exposure to neglect or abuse, children will develop their own psychological "survival" behavior that may or may not go away as the child matures to adulthood.

${ }^{\left[{ }^{14]}\right.}$ A mental illness can be defined as a health condition that changes a person's thinking, feelings, or behavior (or all three) and that causes the person distress and difficulty in functioning.

It is important to remember that the brain is incased in the exoskeleton called a skull bone and is generally inaccessible to confirm or refute theories on its behavior related to mental illness. The only information generally available to medical professionals to know if something is wrong with the brain is the person's behavior. As yet, it has not been possible to model the brain as it acquires mental illnesses or prove any of the theories that cause mental illness proposed. The cause and cure of mental illness has eluded the mental health community.

\subsection{What are the Characteristics of Mental Illness}

The FOFR is essential to all our daily functions in whatever condition it is operating, when a person is faced with a threatening or hostile situation, that the human body use its built-in behavior we call the flight-or-fight response (FOFR). It is initiated deep in the center of the brain where it is well protected and when the conscious refuses for any reason to ignore the demands of the FOFR, mental illness will result.

Mental illness does not occur quickly. The brain does provide many early warning signs that are usually misunderstood, misdiagnosed, overlooked and thus, ignored. The very early warning signs that are usually misunderstood and overlooked and ignored and includes conditions in or on the surface of the body that appear suddenly, and often disappear just as quickly as they appeared and so have been extremely difficult to associate with any one action. These include such physical conditions as chapped lips, psoriasis, acid indigestion/heartburn, hemorrhoids, mouth, lips, cheek and/or tongue sores. These occur when the FOFR decides to reduce the effectiveness of the immune system allowing what foreign bodies that have inhabited the body to go unchecked to some minor degree.

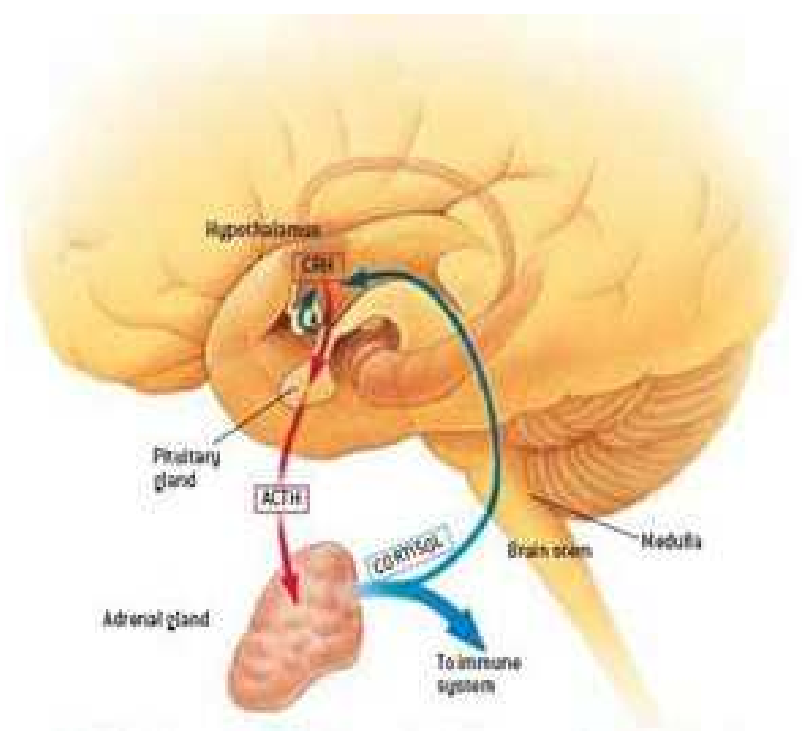

Figure 8. The Brain's FOFR System

${ }^{[15]}$ The key hormone elicited when the FOFR is activated includes adrenaline, also known as epinephrine and neurotransmitters like noradrenalin and norepinephrine. The hormones trigger several systems to prepare the body to face whatever the perceived threats are. The result is increased heart and breathing rate. This serves to ready the body for increased oxygen consumption on the case of flight. Blood is also diverted from the non-essential systems, such as the lymphatic system, stomach and intestines.

Blood vessels direct flow to the muscles required for the fight-or-flight response. The body also releases nutrients 
required to sustain muscular activity. The result is a human body that is set for intense muscular effort. The response continues until the threat is resolved.

${ }^{[16]}$ If a person is placed in a chronically threatening unbalanced environment, the person's FOFR stays activated This constant activation can cause a weakened immune system, an over worked heart, a variety of stomach problems and depression and premature aging. This evidence loosely links stress and depression. Once the threatening situation passes, the brain triggers an adrenaline crash, which triggers the relaxation response.

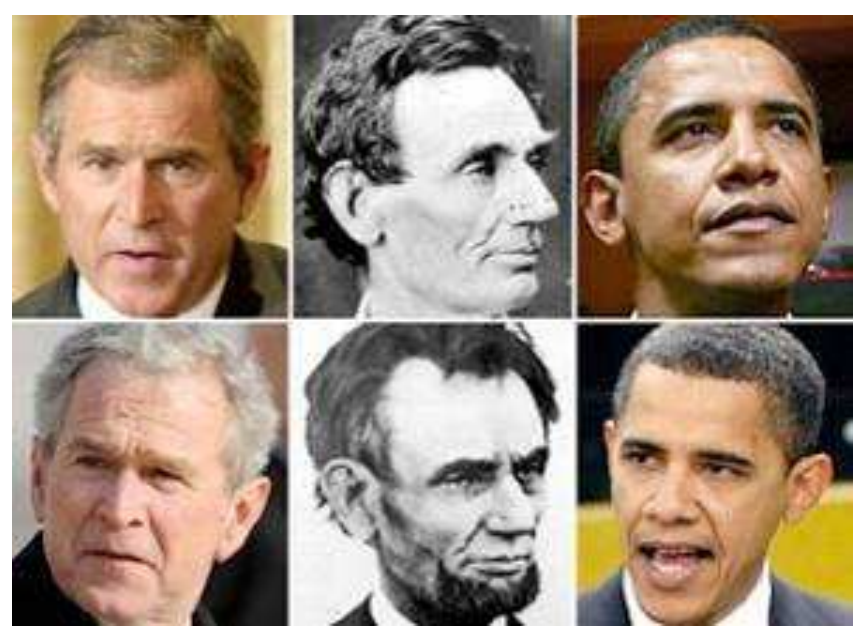

Figure 9. Three Examples of U.S. Presidents that Suffered from Premature aging from the Oversupply of Cortisol and Adrenaline after taking Office. ${ }^{[16]}$

An increase in adrenaline leads to an increase in cortisol. Cortisol is another FOFR hormone linked to the death of all cells that it comes in contact. Brain cells may be destroyed quicker than other cells and are not replaced once damaged. However, parts of the brain neural seem to be repaired. Cortisol can also lower sensitivity to pain, help you to survive grief, or pull you through a short-term pressure project.

${ }^{[16]}$ Unlike adrenaline levels, which decrease rapidly after the stressful situation has passed, cortisol levels increase slowly and decrease slowly. Cortisol stays in the system longer than adrenaline, continually exerting its effect. Cortisol increases blood pressure and blood sugar levels to aid in the flight-or-fight response, but causing premature aging of the body and brain while present.

${ }^{[17]}$ Figure 10 is an image from MRI volumetric studies that illustrating that hippocampal volume is reduced from stress disorders. This atrophy of the hippocampus is believed to cause decreased neuronal density but other studies suggest that the changes are from by whole brain atrophy and generalized white matter atrophy is exhibited by people with stress disorders.

${ }^{18]}$ Figure 11 illustrate the damage (shrinkage) to the brain, frontal lobes and brain stem from long-term exposure to the cortisol and adrenaline released by the brain's FOFR. With much smaller frontal lobes, poor impulse control and decision-making should occur as often does for those with serious mental illnesses.

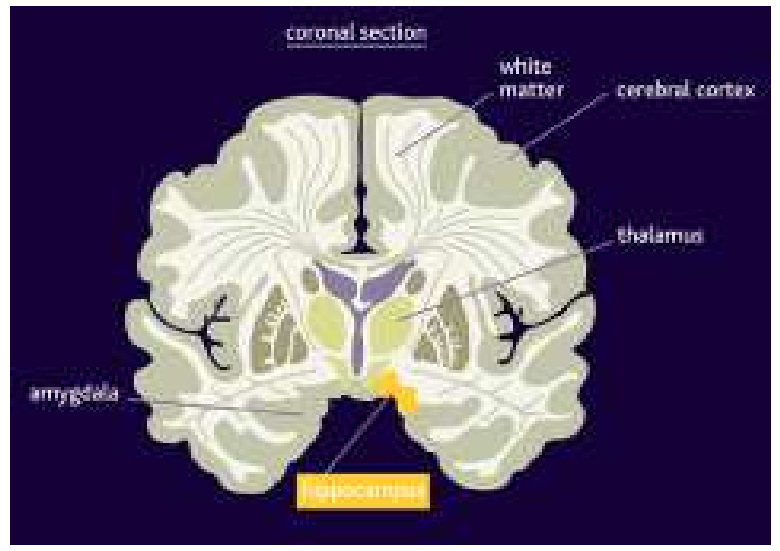

Figure 10. The Atrophy of the Hippocampus, Thalamus and Other Areas of the Brain Occurs when they are Subjected to Long Term Exposure to Adrenaline and Cortisol from Overexposure to Stress such as Occurs in Post Traumatic Stress Disorders (PTSD). ${ }^{[19]}$

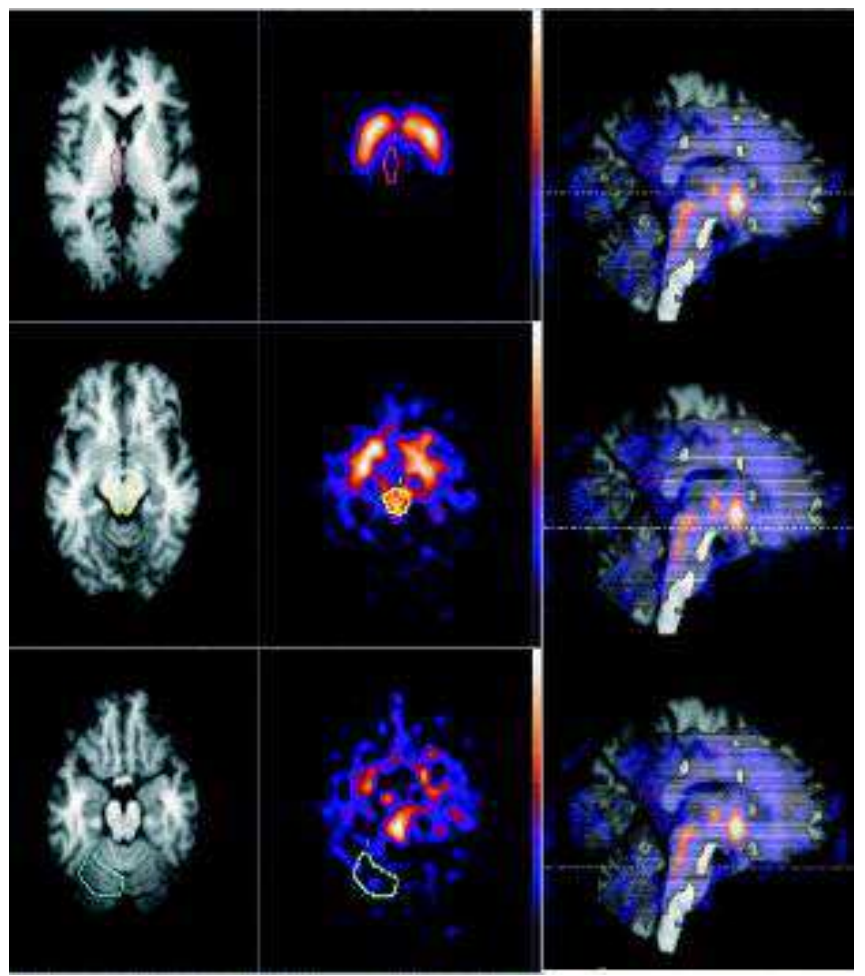

Figure 11. Magnetic Resonance Images (left column), Transversal Slice Single-Photon Emission Computed Tomography (SPECT) (middle column), Midline-Sagittal Co-Registered Images (right side) with Delineation of Thalamic Region (upper row), Midbrain Region (middle row), and Cerebellar Reference Region (bottom row) Illustrating the Degradation of Brain and Brain Stem Tissue as a Result of Damage from the FOFR ${ }^{[18]}$

Degradation and degeneration of the central serotonergic neurotransmission, particularly changes in the presynaptically located serotonin transporter (SERT) availability, are the major mechanisms of neuropsychiatric symptoms. Modern neuro-imaging techniques such as single-photon emission computed tomography and positron emission tomography employ radio labeled tracers, which 
bind to SERT, and thus, allow identification of the decrease in cerebral SERT availability.

The SERT images from patients with depression, anxiety disorders, eating disorders and patients with neurological diseases illustrated damaged tissue from the FOFR. SERT imaging techniques identified the degradation of selective serotonin reuptake-inhibitors from the FOFR. When a person's FOFR is activated continuously, adrenaline and cortisol are secreted constantly at variable levels. This leads, to among other conditions, the increased levels of fatty acids in the blood vessels (cortisol signals the metabolism of fatty acids to produce and emergency supply of energy).

There are many theories on how the high levels of cortisol can cause depression. However, data shows that people who have high levels of cortisol sometimes but not always exhibit symptoms of depression.

Mental illness occurs when the hypothalamus and/or thalamus is degraded or damaged from the presence of cortisol and adrenaline released the FOFR when it is activated, causing premature aging of the body and brain. Normally, the brain maintains the correct chemistry managing the three most influential chemicals associated with normal behavior. These chemicals include serotonin, dopamine and norepinephrine. When the conscious fails to respond to the demands of the FOFR, the FOFR changes the balance between these chemicals, initiating clinical depression.

\subsection{The Definitions of a Threat, Stress, Stressors and Triggers for the FOFR and Why are the Definitions Important}

These terms need to be defined because they often used interchangeably and thus incorrectly. A threat to the FOFR is defined only by the a person's FOFR and is believed to include such events as a dangerous animal ready to strike, a person attempting to strike someone, a person with a gun that may fire the gun to cause harm.

Stress is the response of the body when the FOFR is activated by recognizes that a threat is present. Stressors are those items that activate the FOFR. Indicators that the FOFR has been activated include canker sores in and around the mouth and tongue, chapped lips, heartburn, hemorrhoids (inflamed rectum) and rectal leakage. Stressors are generalized threats such as fellow employees, a bully at school, a failing grade on a test, an angry fried, deadlines, homework, tests, midterms and finals.

Triggers are events, places, people, behavior or items that evoke a psychological reaction. The body responds with a faster heartbeat, faster breathing and increased blood pressure as well as psychological and/or emotional responses.

\subsection{Some Examples of Serious Mental Illnesses}

\subsubsection{Depression}

${ }^{[19]}$ Depression occurs when a person ignores the many messages from the FOFR for a prolong time and the thalamus/hypothalamus area becomes distorted releasing chemicals at levels different than it would if the person was responding properly to the FOFR. It is all too common for people to become insensitive to their feelings and emotions as a survival mechanism from physical and/or psychological trauma. When disassociation with feelings and emotions is used as a survival mechanism, the person's nightly dreams will often be highly emotional, referred to as "unfinished emotional business" from the day or previous day disassociation.

\subsubsection{Major Depression}

Major depression occurs when a person continues to ignore the messages from the subconscious and continues to live in an even more danger environment as determined by a person's FOFR. The subconscious adds more symptoms and more severe symptoms than depression including despair, hopelessness, helplessness and a state of out-of-control. Major depression is the next serious message from the FOFR that the conscious mind is still ignoring the messages from the FOFR and messages from the subconscious. With or without medications for depression, the subconscious/FOFR increases the severity of the symptoms experienced by the conscious mind until the conscious mind must seek help to obtain medications for relief of the severe symptoms.

\subsubsection{Anxiety}

Anxiety is one of many messages from the subconscious mind to the conscious mind that people describe as experiencing a feeing or an emotion. As a survival mechanism by the subconscious, anxiety disorders are a result of the subconscious' determination that immediate danger exists from people, places and activities simultaneously that greatly threatens the FOFR and the person has ignored other messages with the same information in the past regularly. ${ }^{[20]}$ Anxiety is a serious mental illness, one that affects a large number of people. It occurs often and is related to the modern lifestyle that requires people to be exposed continuously to threatening people, places and activities simultaneously on a long term, continuous basis.

\subsubsection{Bi-Polar Disorder}

Bipolar disorder (historically known as manicdepressive disorder) is a psychiatric diagnosis for a mood disorder caused when the FOFR has already used depression and major depression to educate the conscious that it is making too many dangerous decisions and changes the chemistry of brain. The results include causing the conscious large or small swings in a disruptive moods making daily living painful and difficult wanting the conscious to make safer and wiser lifestyle decisions. These encompass a frenzied state known as mania (or hypomania) usually alternated with symptoms of depression. 


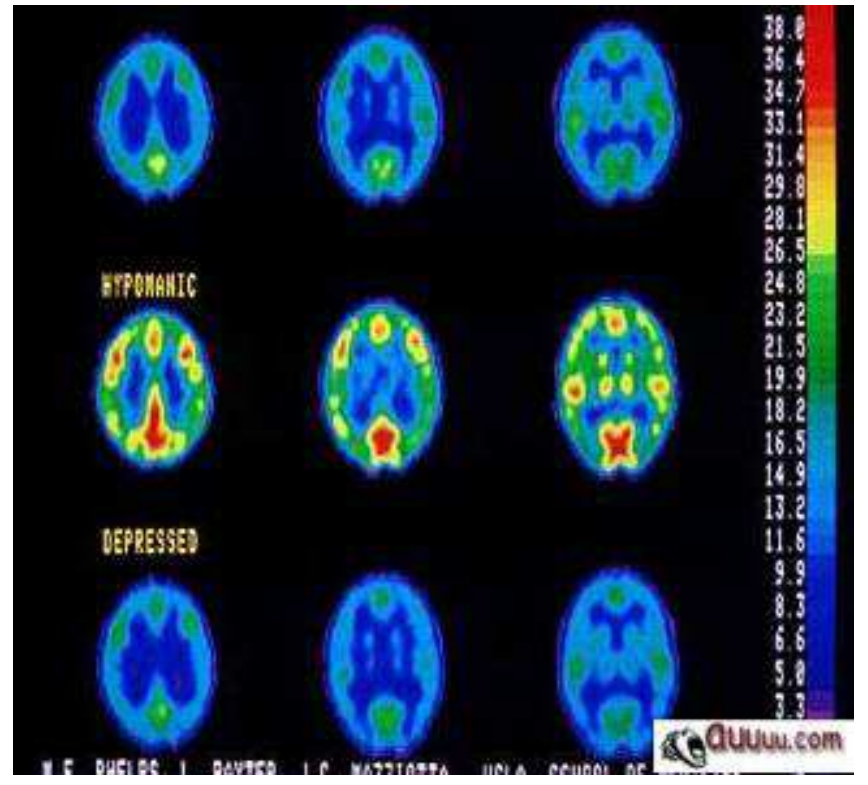

Figure 12. Positron Emission Tomography Scans of the Brain of a Person with Bipolar Disorder. ${ }^{[21]}$

${ }^{[20]}$ Figure 12 illustrates an individual shifting of the brain activity from depression (top row) to mania (middle row) and back to depression (bottom row) over 10 days. Blue and green indicate low brain activity, while red, orange and yellow indicate high brain activity.

As a survival mechanism employed by the subconscious, by the time depression, major depression and anxiety occur from ignoring the subconscious messages from the FOFR, the thalamus/hypothalamus area of the brain has been damaged. The damaged brain keeps the chemistry permanently unbalanced. Without medication, the person with bipolar disorder will eventually have moods swings making them non-functional or experience a psychotic break. This often gets them to a psychiatric hospital and the medications they need to minimize their symptoms.

\subsubsection{Schizophrenia}

Schizophrenia occurs when the FOFR changes the chemistry in the brain causing unrecoverable, self-induced brain damage that causes a failure for internal communications to occur normally. The FOFR does this due to the poor decisions made by the conscious. It is the brains method for ensuring that it can no longer be aware of any real threats and thus stops using the FOFR in a logical way. Schizophrenia is a disorder characterized by a breakdown of thought processes and poor emotional responsiveness. It most commonly manifests itself as auditory hallucinations, paranoid or bizarre delusions, or disorganized speech and thinking, and it is accompanied by significant social or occupational dysfunction.

\section{The Process of Mental Illness}

\subsection{The Fight or Flight Response (FOFR)}

With the redundant sections of the brain used for a variety of functions on a full time or part time basis, the development for self-preservation received the highest priority. Self-preservation is raised to the highest of all functions the brain does.

The brains ability for self-preservation is accomplished by the "fight-or-flight response" (FOFR) and it is invoked every time the general area of the hypothalamus decides a threat either exists or is going to exist soon. The FOFR can control every function of the body; it is the only subsystem to be able to do so.

When the FOFR identifies danger, it activates at least two systems: the sympathetic nervous system and the adrenal-cortical system. The FOFR releases the most powerful and destructive chemicals in the body that are intended to be used for a very brief time to escape danger.

The sympathetic nervous system uses nerve pathways to initiate reactions in the body, and the adrenal-cortical system uses the bloodstream. The combined effects of these two systems implement the desired actions of the FOFR.

${ }^{[21]}$ At the same time, the hypothalamus releases corticotropin-releasing factor (CRF) into the pituitary gland, activating the adrenal-cortical system. The pituitary gland secretes the hormone ACTH (adreno-corticotropic hormone) through the bloodstream and arrives at the adrenal cortex, where it activates the release of approximately 30 different hormones that prepares the body to react to a threat.

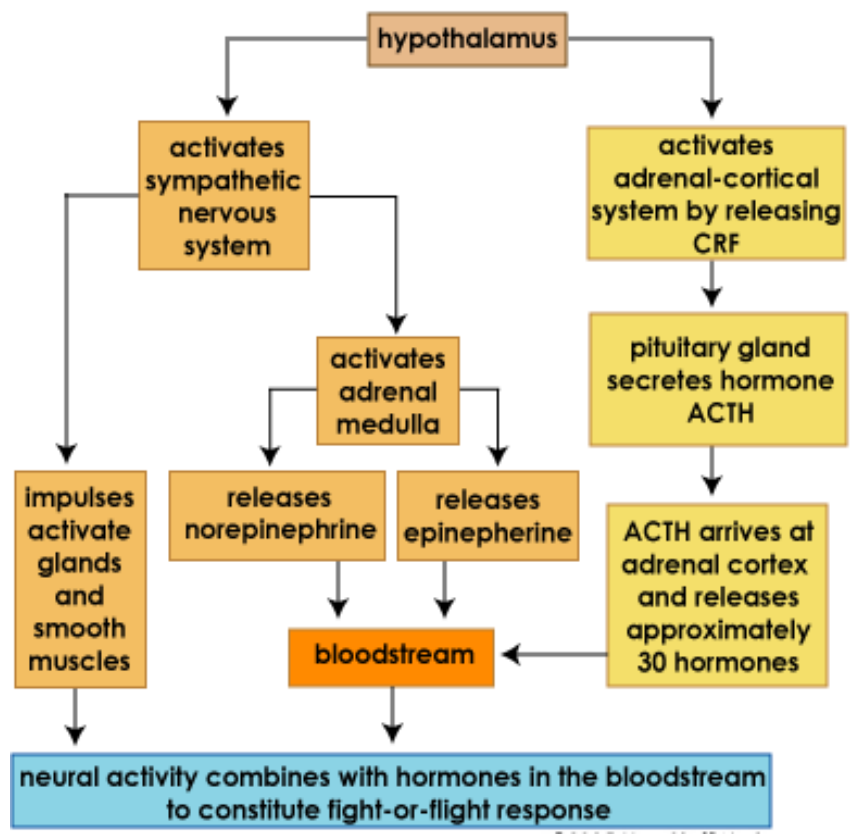

Figure 14. A Theorized Response Triggered by the Fight-or-Flight Responses. $^{[22]}$

The Hypothalamic - Pituitary - Adrenal axis, which is a major neuroendocrine system, relays to the hypothalamus, which secretes "releasing hormones", as they stimulate the pituitary gland to secrete hormones ( a chemical messenger from one cell (or a group of cells) to another. The anterior lobe of the pituitary gland secretes a wide variety of hormones, including "tropic hormones" which stimulate 
other endocrine glands. The adrenal gland releases corticosteroids and catecholamines.

The adrenal medulla: releases the catecholamine adrenalin. The adrenal cortex zona glomerulos secretes mineralocorticoids, mainly aldosterone. The zona fasciculata secretes corticosteroids, among them cortisol, a hormone that regulates every organ and tissue function. The zona reticularis: secretes androgens such as testosterone.

The adrenal medulla: releases the catecholamine adrenalin. The adrenal cortex zona glomerulos secretes mineralocorticoids, mainly aldosterone. The zona fasciculata secretes corticosteroids, among them cortisol, a hormone that regulates every organ and tissue function. The zona reticularis: secretes androgens such as testosterone.

The hypothalamus secretes corticotropin releasing hormone and arginine vasopressin, which stimulate the anterior lobe of the pituitary gland to release adrenocorticotropic hormone or ACTH. ACTH causes the adrenal cortex to release glucocorticoids, primarily cortisol which enhances metabolism in a number of ways. Cortisol stimulates the release of amino acids from the body and stimulates lipolysis or the breakdown of fat.

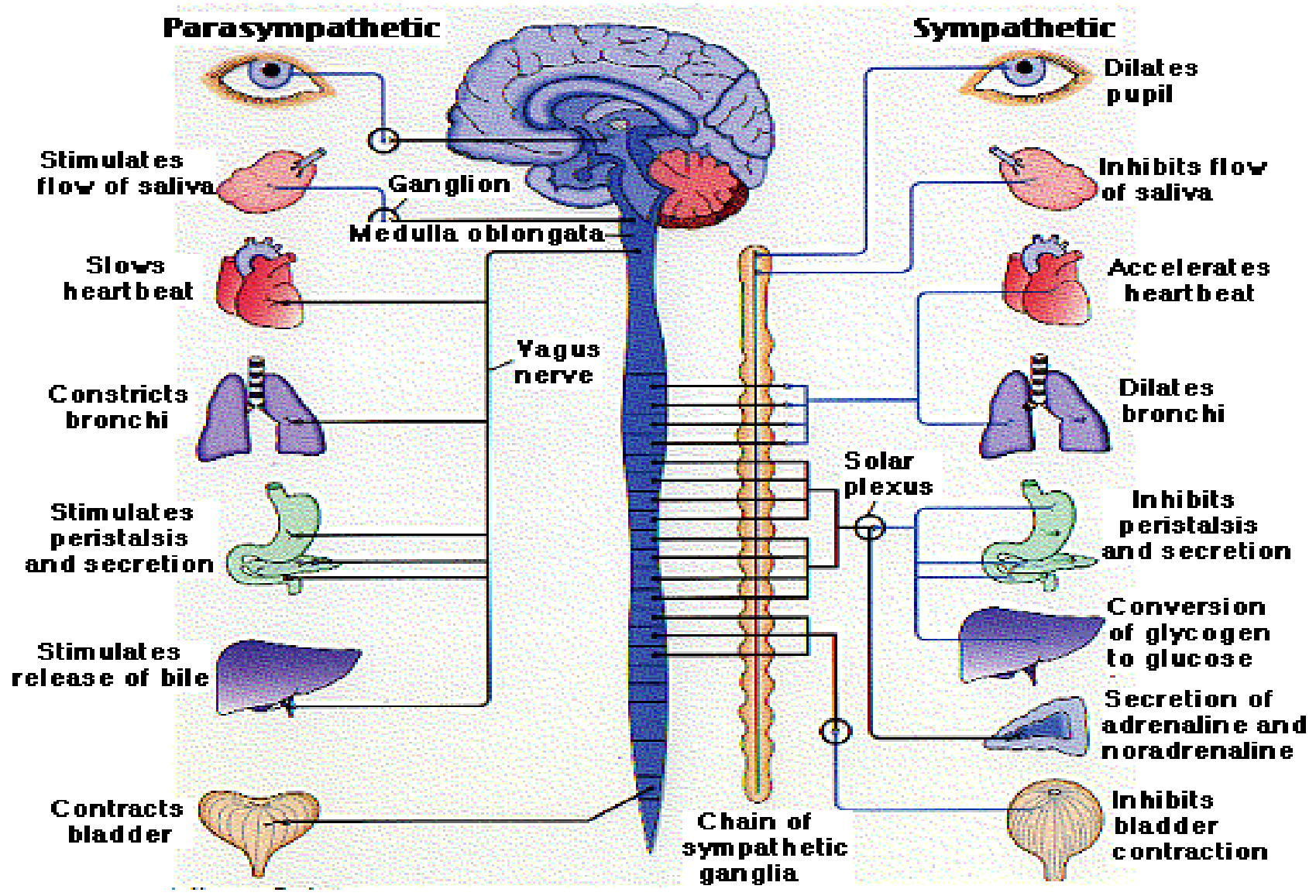

Figure 13. Some of the Many Parts of the Body that the FOFR Controls Continuously for our Self-Preservation Demonstrating that it is the FOFR that Must be Responded to Maintain Good Mental and Physical Health.

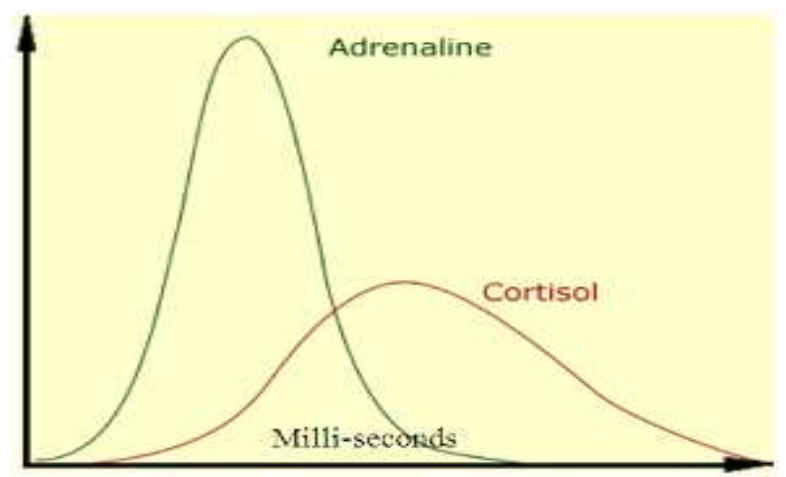

Figure 15. Normal Adrenaline and Cortisol Levels in the Human Body for the Activated FOFR.

It stimulates the release of gluconeogenesis from the newly released amino acids and fatty acids that conserve glucose by inhibiting its uptake into muscle and fat cells. It releases cortisol, increasing blood sugar. It forces a rise in blood pressure and suppresses the immune system and suppressing inflammation. In summary, the overall effect is a person that can run and react faster with more endurance, see better due to the dilation of the pupils, hear more acutely and think faster than it could before the cascade of reactions. ACTH causes the adrenal cortex to release Excess waste may be voided and food present in the stomach may be vomited to be lighter. However, all bodily functions not needed for the immediate survival are shut down such as digestion, reproduction and immunity. This is where chronic FOFR or stress of long duration begins to have a deleterious effect leading to high blood pressure and 
type 2 diabetes

Both of these may result in arteriosclerosis leading in turn to heart disease, stroke and premature death. Inflammation is suppressed by preventing phospholipid release, decreasing eosinophil action and various other mechanisms.initiated. This determination is made well outside the awareness of conscious at the subconscious level.

However, the subconscious does provide many messages to the conscious to be aware of, but today they are usually misunderstood and misdiagnosed, thus ensuring that that the symptoms of mental illness will continue to increase. Some symptoms caused from living an unbalanced lifestyle include long-term chapped lips, psoriasis, a continuous stream of colds and flues as the immune system is suppressed by the FOFR. Also bacterial infections in the throat, migraine headaches, acne, mouth and lip sores, difficulty sleeping, getting to sleep and staying asleep, difficulty concentrating, difficulty with memory, restless legs syndrome, excessive smoking, over use of alcohol and illegal and illicit drugs, drug dependency, conflicts with family and friends and general irritability.

Some symptoms include long-term chapped lips, psoriasis, a continuous stream of colds and flues as the immune system is suppressed by the FOFR. Also bacterial infections in the throat, migraine headaches, acne, mouth and lip sores, difficulty sleeping, getting to sleep and staying asleep, difficulty concentrating, difficulty with memory, restless legs syndrome, excessive smoking, over use of alcohol and illegal and illicit drugs, drug dependency, conflicts with family and friends and general irritability. Other symptoms include difficulty understanding other people's behavior, believing everyone else is problematic, refusing to seek professional counseling services choosing to believe that mental illness can be cured using vitamins and supplements, refusal to attend spiritual services and arguing in groups of people and friends.

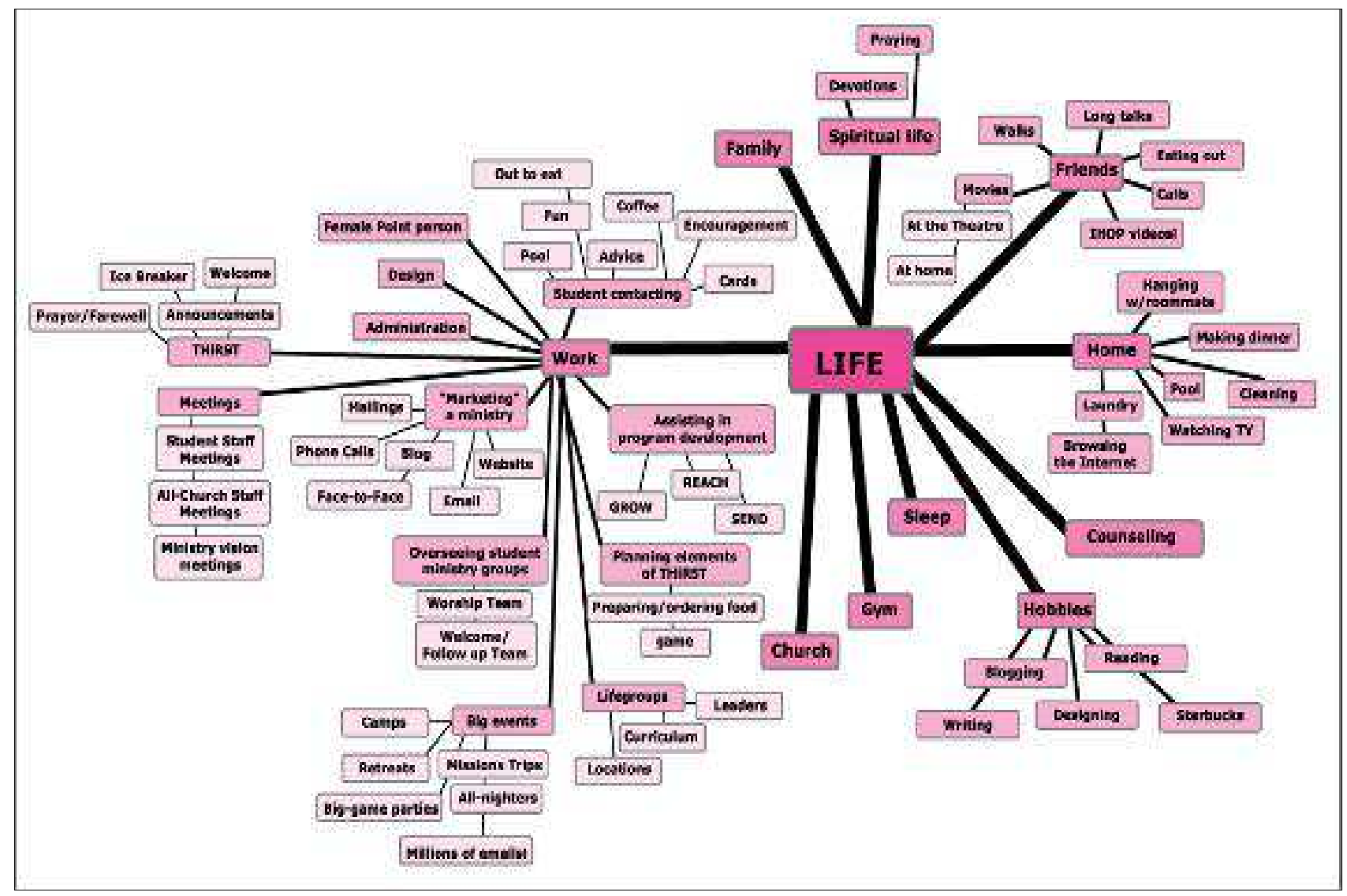

Figure 16. An Example of a Person's Unbalanced Lifestyle, Created by the Conscious over Many Years by Addressing only the Needs of the Conscious

These symptoms indicate that the subconscious detects a threat(s) and requires action to be taken by the conscious. Unfortunately the subconscious does not indicate the conscious what action needs to be taken other than eliminate the presence of the threat. The person should be feeling fear if they are sensitive to their feelings and "feeling" out-of-control and "powerless" to change circumstances and the FOFR wants the conscious to change.

\section{Predicting Mental Illness}

\subsection{The Processing of the Information from the Circadian and Ultradian Rhythms using Predictive Algorithms}

${ }^{[23]}$ Figure 17 is the proprietary results of a prognostic 
analysis completed using data-driven predictive algorithms to process the time-series equipment analog telemetry from three NASA satellite gyroscope units A, B \& C recorded and stored from January 2, 1993 to February 24, 1993. The processed analog motor current telemetry from Gyro A and $\mathrm{C}$ do not have any premature aging present. The behavior of the telemetry is uniform. The telemetry from Gyro B does illustrate the presence of a non-repeatable transient event (NRTE) also caused from the premature aging of at least one part in the Gyro. The NRTE is caused from the unpredictable interaction of the part with premature aging with the other parts with different aging behavior.
A prognostic analysis processes normal appearing data with predictive algorithms so that any premature aging will be illustrated. Predictive algorithms are necessary because of the belief that equipment failures were instantaneous and random (this is known as having the Markov property) and unrelated to any previous behavior in the data. Premature aging was not recognized to exist in electrical and mechanical engineering due to the use of the Markov property used in probability analysis and so it was misdiagnosed and overlooked as systemic noise when present in the space industry.

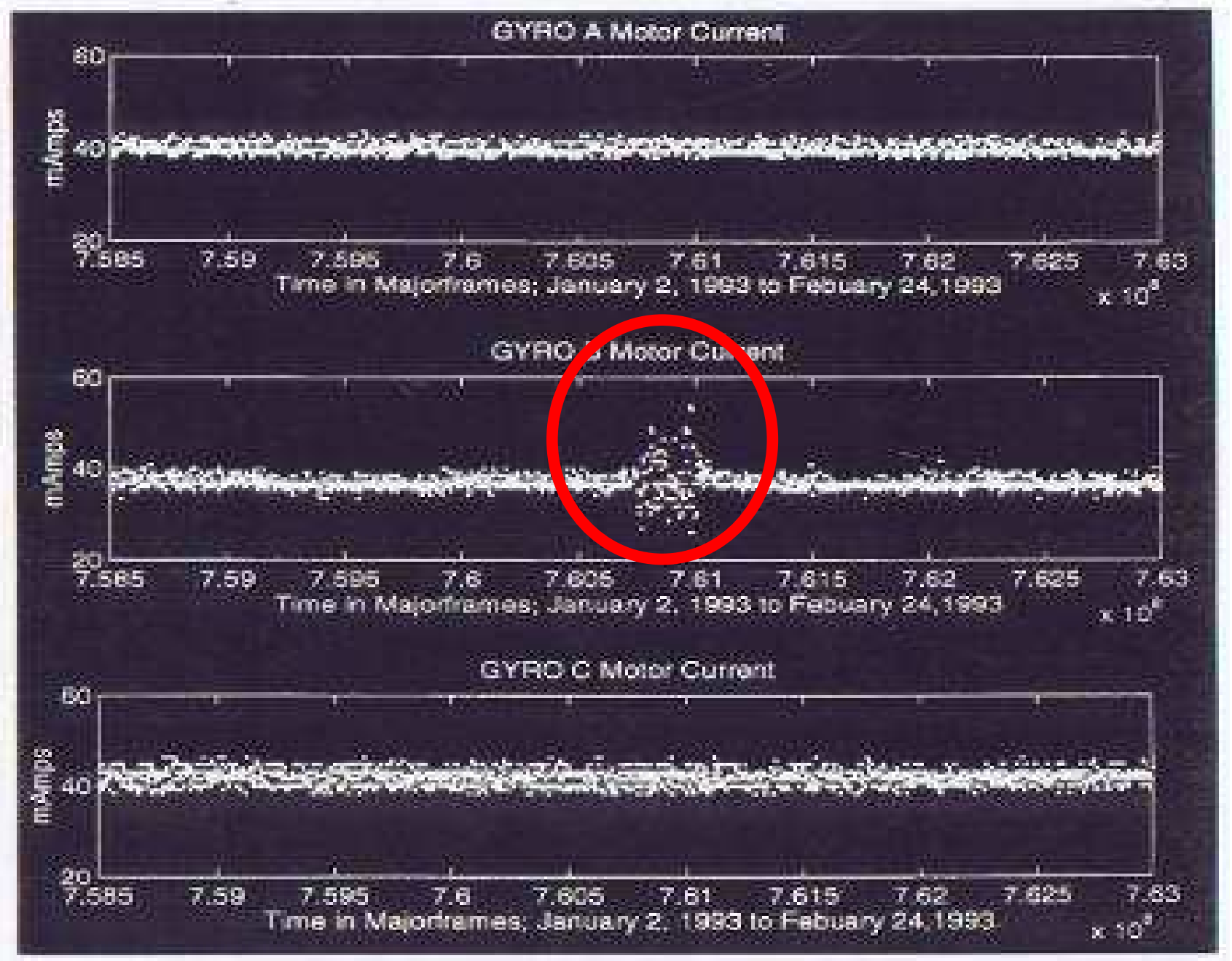

Figure 17. An Example of the Post-Processing Results using Proprietary, Predictive Algorithms to Illustrate Premature Aging (a.k.a. non-repeatable transient Event) Circled in Red after Processing Normal Appearing Telemetry from Fully Functional Satellite Gyroscope Telemetry Data. ${ }^{[34]}$

Probability analysis requires any behavior it is used with to quantify in probabilities to be instantaneous and random (a.k.a. Markov property) whether it is or not. Probability analysis has been used to quantify equipment reliability in the space industry for more than 50 years and engineers soon believed that equipment failures were instantaneous and random and could not be predicted nor prevented. Predictive science was developed to replace probability analysis to quantify equipment reliability with certainty rather than probabilities.
The horizontal scale is proprietary. The three satellite rate-gyros were used on the NASA extreme, ultra violet explorer (EUVE) satellite, a sister satellite to the NASA Hubble satellite that locates targets in visible light frequencies.

The EUVE satellite was located at a 275-mile orbit altitude at the time the prognostic analysis was completed. The NASA EUVE satellite re-entered the Earth's atmosphere and crashed in an Egyptian desert in 2002. The rate-gyros are used for satellite attitude control, 
satellite/telescope pointing and reorienting of the satellite and telescope to EUV targets of interest. The time that the equipment telemetry spanned in the prognostic analysis in Figure 17 is equivalent to that of a prelaunch readiness, launch and 30 days of on-orbit history for a human space flight program.

${ }^{[13]}$ The prognostic analysis completed on the NASA EUVE satellite's gyro motor current analog telemetry in Figure 17 illustrates that all three rate-gyros were operating simultaneously for redundancy purposes, however only two gyros were needed to maintain satellite attitude. The nonrepeatable transient event (NRTE) (a.k.a. premature aging) illustrated in red in the Gyro B telemetry motor current was observable only after processing the rate gyro analog telemetry using proprietary data-driven predictive algorithms.

The same telemetry data before processing with predictive algorithms were evaluated by the engineers that designed and tested the rate gyros prior to launch and they did not observe any unusual behavior in the telemetry. The predictive algorithms were pioneered on the Air Force's GPS satellites telemetry in 1980 to predict GPS satellite atomic clock failures during system wide GPS testing. The Gyro B unit failed when the data stops. The period from the NRTE in the Gyro B telemetry to the end of the data was the duration of remaining life predicted for Gyro B using a prednostic analysis.

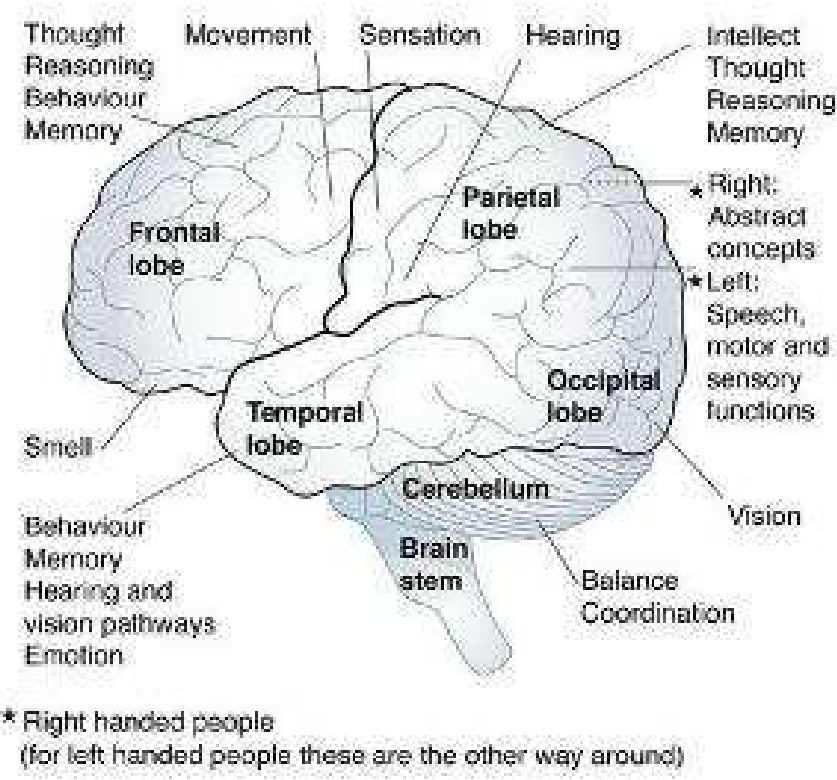

Figure 18. The Subsystems of the Human Brain and their Expected Functionality.

In electrical communication theory, modulation techniques add intelligence onto an electrical or RF carrier signal. Demodulation recognizes the presence of modulation on a carrier and demodulates the information.

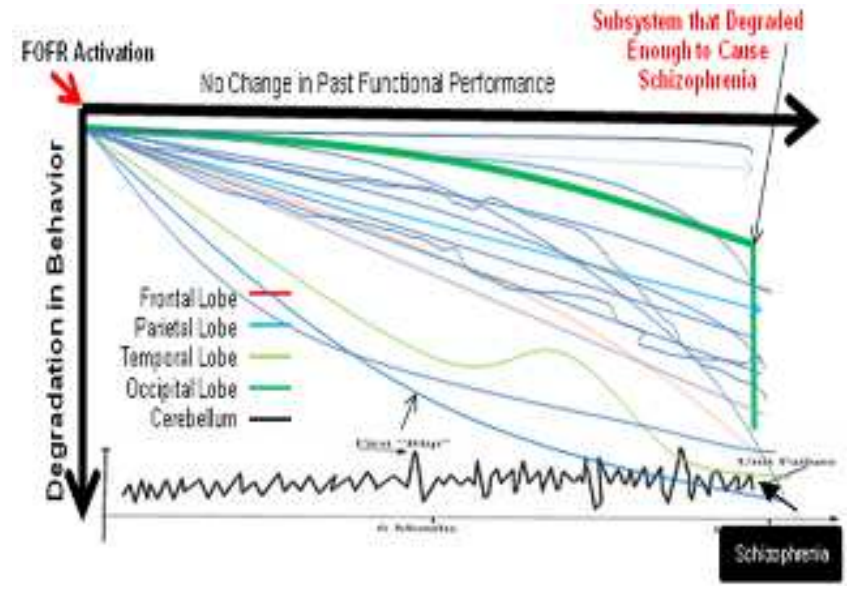

Figure 19. An Example of the Different Rates of Degradation in Aging for the Brains Subsystems caused by Cortisol and Adrenaline Released by the FOFR, Eventually Resulting in Mental Illness.

\subsection{How Damage to the Body and Brain Occurs when the FOFR Remains Activated}

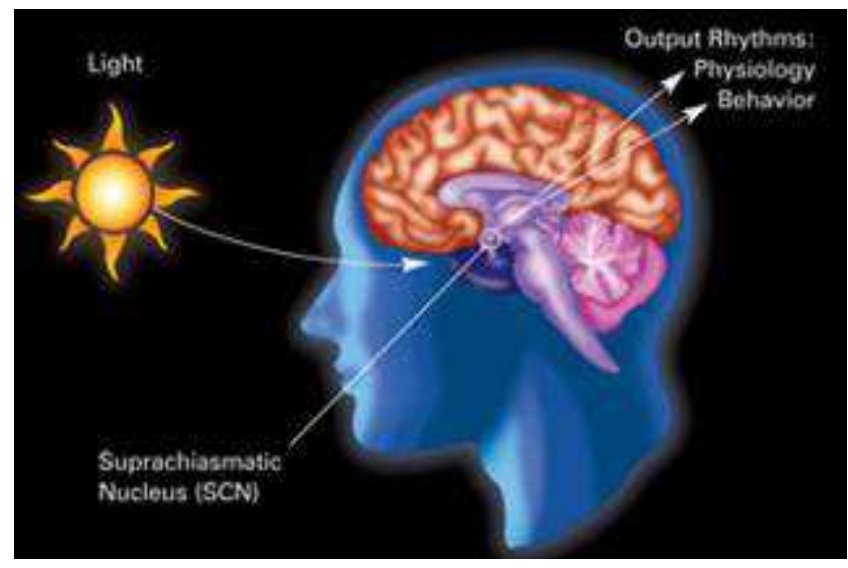

Figure 20. The Location in the Brain where Circadian and Ultranian Rhythms Originate Near the Thalamus and Hypothalamus that Create the FOFR that are Damaged from Long Term Exposure to Cortisol and Adrenaline.

This different rate of degradation for the brain's subsystems (see Figure 19) that is caused from the premature aging by the chemicals released by the FOFR long-term is the basis for the application and usability of the prognostic and health management program to predict and prevent mental illness. As the brain's subsystems degrade in performance, their functionality changes and this change in functionality is illustrated in telemetry, using predictive algorithms in a PHM.

\subsection{The Data Bandwidth between the Subconscious and the Conscious}

Using feelings, hunches and emotions to communicate from the subconscious to the conscious mind, the information input to the conscious mind from the subconscious mind is significantly less than the sensory input into the FOFR subsystem. The conscious mind would be easily overloaded if it received all the information that 
the FOFR system received from a person's eyes, ears, nervous system and skin and nose and so it is estimated that less than $1 \%$ of the data that the subconscious receives is sent to the conscious to create our reality.

\subsection{The Method for Predicting Mental Illness from Information taken from Astronauts Circadian and Ultradian Rhythms}

Mental illness can be predicted by using predictive algorithms to process and display the information collected from a person that is related to the circadian and ultradian rhythms. The predictive algorithms will illustrate the presence of premature aging, identifying an offset or nonrepeatable transient event (a.k.a. premature aging) exists for the personnel on board space exploration missions. The offset is identified by comparing a person's baseline Circadian and Ultradian rhythms behavior and real-time Circadian and Ultradian rhythms. If either or both exists, it means that the FOFR/thalamus is degraded by the harsh chemicals of the FOFR and the thalamus will continue to degrade in performance enough to cause mental illness in the near future.

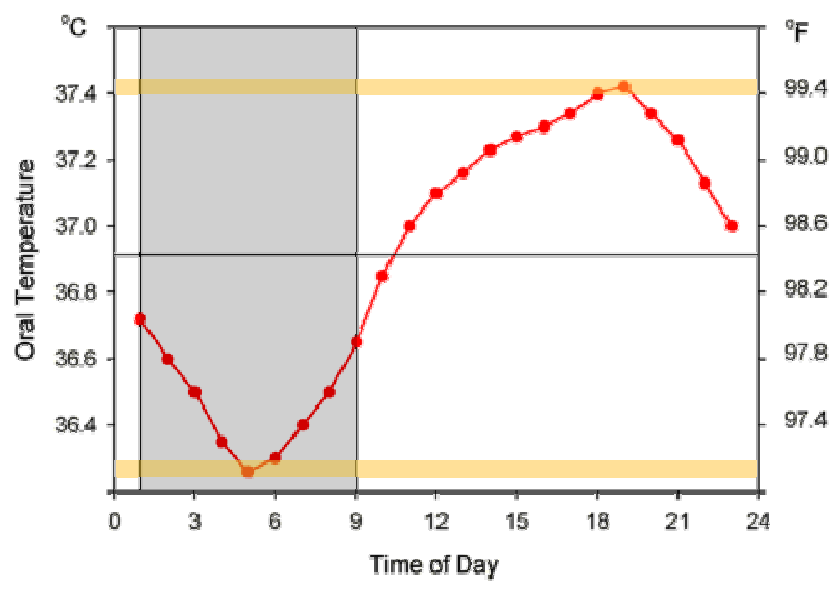

Figure 21. An Example of a Person's Normal Short-Term Body Temperature for Developing a Baseline Body Temperature Behavior for Comparison with the Behavior after an Astronaut is in Deep Space

${ }^{[11]}$ Predictive algorithms require information from the inside of the active part of a system. Fortunately, the part of the brain that controls Circadian rhythms resides next to the thalamus and hypothalamus and the behavior that is controlled by the Circadian rhythm proved the information to be processed using predictive algorithms.

${ }^{[24]}$ Almost every function in the human body exhibits a Circadian rhythm. In ambulatory conditions, environmental factors and physical exertion can obscure or enhance the expressed rhythms. The three most commonly monitored vital signs are blood pressure (systolic and diastolic), heart rate and body temperature. Because these variables exhibit daily rhythms, their normal values vary with the time of day.

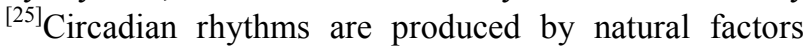
within the body, but they are also affected by signals from the environment. Light is the main cue that triggers
Circadian rhythms, and turning light on or turning light off trigger the genes that control an organism's internal clocks. Circadian rhythms are physical, mental and behavioral changes that follow an approximate 24-hour cycle, responding primarily to the light and darkness in a person's immediate environment. They are found in people, most living things, including animals, plants and many tiny microbes.

${ }^{[26]}$ Ultradian rhythms play an essential part at all levels of biological organization, providing timekeeping for intracellular processes, playing various roles in intracellular signaling systems and underpinning coherent behavior in tissues and organs. They are crucial to endocrine and neural performance and in psychobiology. The body's master clock controls the production of melatonin, a hormone for sleep. The area of the brain that creates Circadian rhythms is located just above the optic nerves, which relay information from the eyes to the brain and is the area receives information about incoming light. When there is less light-like at night - the area tells the brain to make more melatonin so a person gets drowsy.

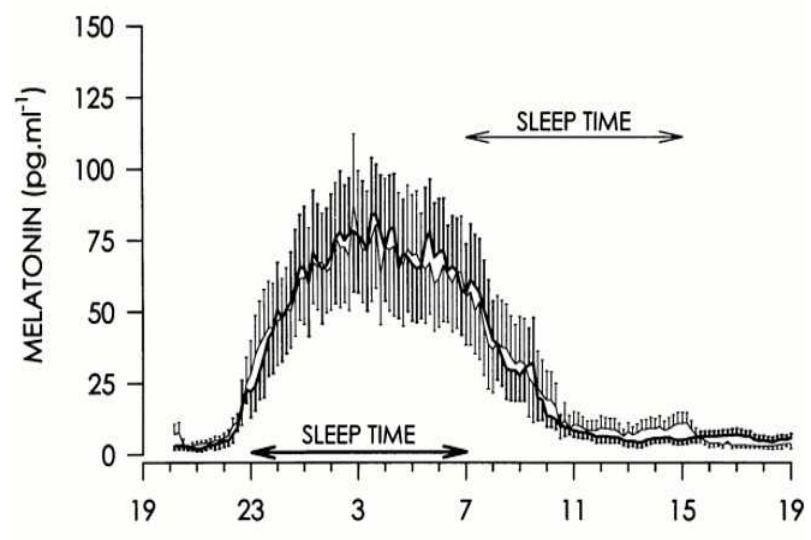

Figure 22. A Example of a Person's Normal Short-Term Circadian Rhythms from Telemetry for Creating a Normal Baseline

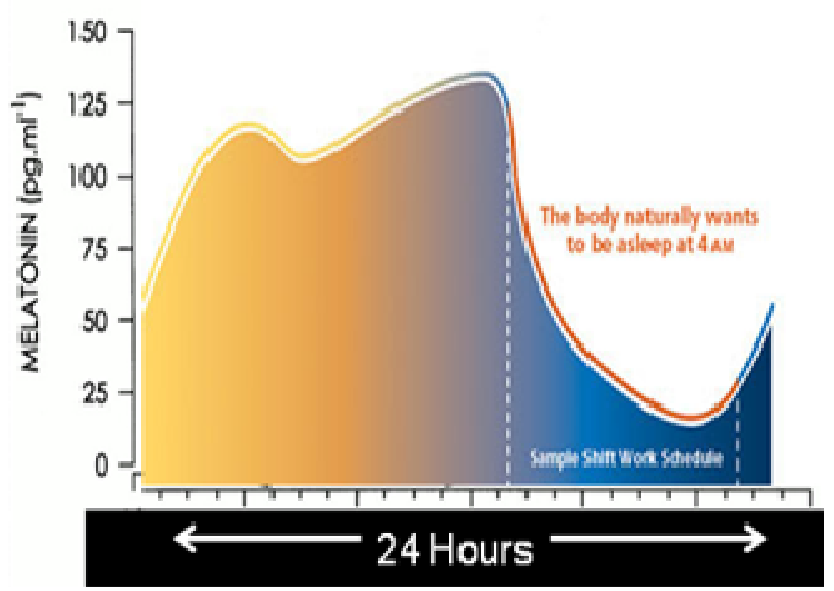

Figure 23. An Example of the Gross Change to a Person's Circadian Rhythm as Compared to Figure 22 Caused from too much Adrenaline and Cortisol Preceding the Onset of Mental Illness ${ }^{[5]}$ 
Ultradian rhythms control events in the body that are repeated throughout a 24-hour circadian day. In contrast, infradian rhythms, are events such as the menstrual cycle, have period longer than a day.

\subsection{The Biological, Physiological and Engineering Diagnostic Information Needed in Telemetry from Astronauts to Predict, Prevent and Recover from Mental Illness}

${ }^{[4]}$ Before being launched to space, the rate the biological information needed to develop a baseline behavior is much higher than after the person arrives in space and works with a daily routine. This is because the time in preparation for launching to space may be very low (a few weeks to a few months) while the time in space may be very long. If the preparation period will be less than several weeks then sampling frequency for each measurement should be continuous or as continuous as feasible. If the preparation period for launch will occur over many weeks, then the sampling frequency can be reduced to once/hour.

In general, the shorter the preparation time for launch (a.k.a. prelaunch readiness), the higher the data sampling frequency is required. Preferably, prelaunch activity biological, physiological and engineering diagnostic data should be recorded continuously and the data available for merging with launch and on-orbit data. After a person initially arrives in space, the sampling frequency should be continuous or near continuous as possible. After the person has been in space for several weeks, the sampling frequency can be reduced to once/hour. If a person is in space for several months, the sampling frequency can be reduced to as little as once/ 6 hours.

Table 2 defines the diagnostic telemetry measurement sampling rates needed to predict mental illness from each of the different phases that data is generated and made available for processing for completing prognostic analysis during space exploration. This information will be used to create a baseline behavior for comparison with actual data to discriminate between normal and abnormal behavior.

The physiological measurement range is important to define early in the design phase of the vehicle the astronauts will be living in. The data acquisition system often use $5^{\text {th }}$ and $6^{\text {th }}$ order polynomials to convert the original analog data that's been converted to digital data for transmitting to the ground and back to analog data so the telemetry processing and display system can accurately relay the biological, diagnostic information to ground personnel for analysis.

Table 2. The Sampling Rates For Diagnostic Data for Processing with Predictive Algorithms used by Ground Personnel to Process with Predictive Algorithms to Illustrate the Presence of Accelerated Aging and Predict Mental Illness in Astronauts in Deep Space. ${ }^{[4]}$

\begin{tabular}{llll}
\hline During Launch Readiness & During Launch & After 6 Weeks in Space & After 12 Weeks in Space \\
\hline \multirow{2}{*}{ Continuous or Near Continuous Data } & Continuous or Near Continuous & 1 Sample/ \\
& Data & hour & 1 Sample/6 hours \\
\hline
\end{tabular}

Table 3. Examples of Biological and Physiological Diagnostic Measurements from an Astronaut in Space used to Predict, Prevent and Cure Mental Illness in a Predictive Medicine Program using the Predictive Algorithms in Table ${ }^{\text {[4] }}$

\begin{tabular}{|c|c|c|c|}
\hline Measurements & Measurement Sample Rate & 8-Bit LSB Value & Measur-ment Range \\
\hline Systolic BP (Wide) & At least once/day & $0.01(\mathrm{~mm} \mathrm{Hg})$ & $30-200$ \\
\hline Systolic BP (Narrow) & At least once/day & $0.01 \mathrm{~mm} \mathrm{Hg}$ & $50-150$ \\
\hline Diastolic BP (Wide) & At least once/day & $0.01(\mathrm{~mm} \mathrm{Hg})$ & $30-200$ \\
\hline Diastolic BP (Narrow) & At least once/day & $0.01 \mathrm{~mm} \mathrm{Hg}$ & $75-150$ \\
\hline Heart Rate Wide Range & At least once/day & 0.1 (bpm) & $0-300$ \\
\hline Heart Rate Narrow Range & At least once/day & $0.1 \mathrm{bpm}$ & $50-100$ \\
\hline Temperature & At least once/day & $0.01\left({ }^{\circ} \mathrm{C}\right)$ & $95-105$ \\
\hline Melatonin Level & At least once/day & $1.0 \mathrm{pg} / \mathrm{ml}$ & $0-100$ \\
\hline Cortisol Level & At least once/day & $0.1 \mathrm{mcg} / \mathrm{dl}$ & $0-60$ \\
\hline Subjective Alertness & At least once/day & $1.0 \mathrm{~mm}$ & $0-100$ \\
\hline Desired Liquid Intake & At least once/day & $1.0 \mathrm{ml}$ & $0-2 \mathrm{~L}$ \\
\hline Desired Solid Food Intake & At least once/day & $1.0 \mathrm{~g}$ & $0-5 \mathrm{~kg}$ \\
\hline Required Solid Food Intake & At least once/day & $1.0 \mathrm{~g}$ & $0-3 \mathrm{~kg}$ \\
\hline Required Liquid Intake & At least once/day & $1.0 \mathrm{~g}$ & $0-2 \mathrm{~L}$ \\
\hline Actual Liquid Intake & At least once/day & $1.0 \mathrm{~g}$ & $0-2 \mathrm{~L}$ \\
\hline Actual Solid Food Intake & At least once/day & $1.0 \mathrm{~g}$ & $0-3 \mathrm{~kg}$ \\
\hline Actual Liquid Waste & At least once/day & $1.0 \mathrm{~g}$ & $0-2 \mathrm{~L}$ \\
\hline Actual Solid Waste & At least once/day & $1.0 \mathrm{~g}$ & $0-3 \mathrm{~kg}$ \\
\hline Duration of Sleep/Day & At least once/day & $5 \mathrm{~min}$ & $0-1500$ \\
\hline Duration of Rest/Day & At least once/day & $5 \mathrm{~min}$ & $0-1500$ \\
\hline Duration of Recreational Activities/day & At least once/day & $5 \mathrm{~min}$ & $0-1500$ \\
\hline Duration of Work/Day & At least once/day & $5 \mathrm{~min}$ & $0-1500$ \\
\hline
\end{tabular}




\begin{tabular}{llll}
\hline Measurements & Measurement Sample Rate & 8-Bit LSB Value & Measur-ment Range \\
\hline Duration of REM Sleep & At least once/day & $1 \mathrm{~min}$ & $0-1200$ \\
Duration of Non-REM Sleep & At least once/day & $5 \mathrm{~min}$ & $0-1500$ \\
Frequency of Urination & At least once/day & 1 & $0-40$ \\
Frequency of Emptying Bowel & At least once/day & 1 & $0-15$ \\
Duration Between Urination & At least once/day & 1 & $0-1500$ \\
Duration Between Emptying Bowel & At least once/day & 1 & $0-6000$ \\
Unsuccessful Urinations & At least once/day & 1 & $0-25$ \\
Unsuccessful Emptying of Bowel & At least once/day & 1 & $0-25$ \\
Duration of Desired Physical Activity/Day & At least once/day & $1 \mathrm{~min}$ & $0-600$ \\
Duration of Required Physical Activity/Day & At least once/day & $1 \mathrm{~min}$ & $0-600$ \\
Duration of Physical Activity & At least once/day & $1 \mathrm{~min}$ & $0-600$ \\
Duration of No Activity & once/day & $1 \mathrm{~min}$ & $0-6000$ \\
Duration of No Required Activity & once/day & $1 \mathrm{~min}$ & $0-6000$ \\
\hline
\end{tabular}

\section{Recovering from Mental Illness}

\subsection{Recognizing and Responding to the Messages from the FOFR is not Optional}

The complexity of today's living conditions keeps people in a high-stressed, unbalanced life. The unbalanced lifestyle is a result of the migration of people to the city from urban or rural areas. In today's daily life, people are forced to return to situations that the person's unconscious determines to be threatening. This can occur at the person's place of employment, in their relationship with their spouse or significant other or social group. The unbalanced lifestyle is the prominent lifestyle in today's society.

It is when the person's subconscious determines that its conscious is in situations that cause the FOFR to be out-ofcontrol and/or powerless to escape its perceived threat that mental illness may be initiated. This determination is made well outside the awareness of conscious at the subconscious level. However, the subconscious does provide many messages to the conscious, symptoms for the conscious to be aware of, but today they are misunderstood and misdiagnosed, thus ensuring that that the symptoms of mental illness will continue to increase.

Some symptoms of stress caused from living an unbalanced lifestyle include long-term chapped lips, psoriasis, a continuous stream of colds and flues as the immune system is suppressed by the FOFR. Also bacterial infections in the throat, migraine headaches, acne, mouth and lip sores, difficulty sleeping, getting to sleep and staying asleep, difficulty concentrating, difficulty with memory, restless legs syndrome, excessive smoking, over use of alcohol and illegal and illicit drugs, drug dependency, conflicts with family and friends and general irritability. Other symptoms include difficulty understanding other people's behavior, believing everyone else is problematic, refusing to seek professional counseling services choosing to believe that mental illness can be cured using vitamins and supplements, refusal to attend spiritual services and arguing in groups of people and friends. These symptoms indicate that the subconscious detects a threat(s) and requires action to be taken by the conscious. Unfortunately the subconscious does not indicate the conscious what action needs to be taken other than eliminate the presence of the threat. The person should be feeling fear if they are sensitive to their feelings and "feeling" out-of-control and "powerless" to change circumstances and the FOFR wants the conscious to change.

\subsection{Important Factors for Recovering from Mental Illness}

${ }^{[5]}$ Ultradian rhythms also control human movements, sleep, attention span and alertness and so should be used to start evaluating the decisions being made.

The onset of mental illness can be detected by recognizing the presence of minor changes to the Circadian rhythms (premature aging) that are caused by the lack of the following items in a person's life.

- Recognizing that the subconscious is providing warnings as depression and major depression and learn the behavior necessary to stop the progression to bi-polar and/or schizophrenia

- Recognizing feelings and emotions as messages from the subconscious mind to the conscious mind

- Eliminate the threat(s) that the FOFR identifies is/are present.

- Eliminate the presence of the harsh chemicals released by the FOFR from the body by ensuring a life style that does not require the FOFR to be utilized.

- Eliminate the presence of all behavior that can be identified as a threat by the FOFR

- Allow the hypothalamus the time to heal from the damage caused by the presence of the harsh chemicals released by the FOFR

- Develop a balanced lifestyle that includes:

- $\quad$ Making and keeping a large number of friends

- A strong bond with family and ex-family members

- Fun

- Work/employment

- Religion, daily recognition of a higher power

- $\quad$ Spirituality including praying out load (so that the ears can be used to communicate with the 
subconscious mind)

- $\quad$ Play/participate in a sport(s) program

- Initiate artistic endeavors

- Start aerobic exercise including:

- Maintaining healthy body weight by eating a diet with portions of meat, fish, poultry, vegetables, fruits, nuts, simple and complex carbohydrates

- Vitamin and mineral supplements

- Medications as prescribed by medical doctors

- Periodic visits to psychologist or psychotherapist for maintaining high self awareness

- A self-defense program. The subconscious mind (a person's self-protection system) responds in many positive ways when the conscious mind decides to train the physical body to protect itself.

- A relaxation program.

\subsection{Developing and Keeping a Balanced Lifestyle}

A balanced lifestyle includes the essential elements of our life in the equal proportion. Adopting a balanced lifestyle is of primordial importance because it has immediate and long-term effects on our mental and physical health. We become ill most often under stress (threats) because the first action that the FOFR does when it is activated at any level is suppress or turn down our immune system. It does this in the short period that the FOFR was to be active, and during this brief period, our immune system can function without assistance. While the immune system is suppressed long term by the FOFR, people can expect to get cancers, heart failure, organ failures, stroke, diabetes and any of the chronic diseases that our immune system normally stops from occurring.

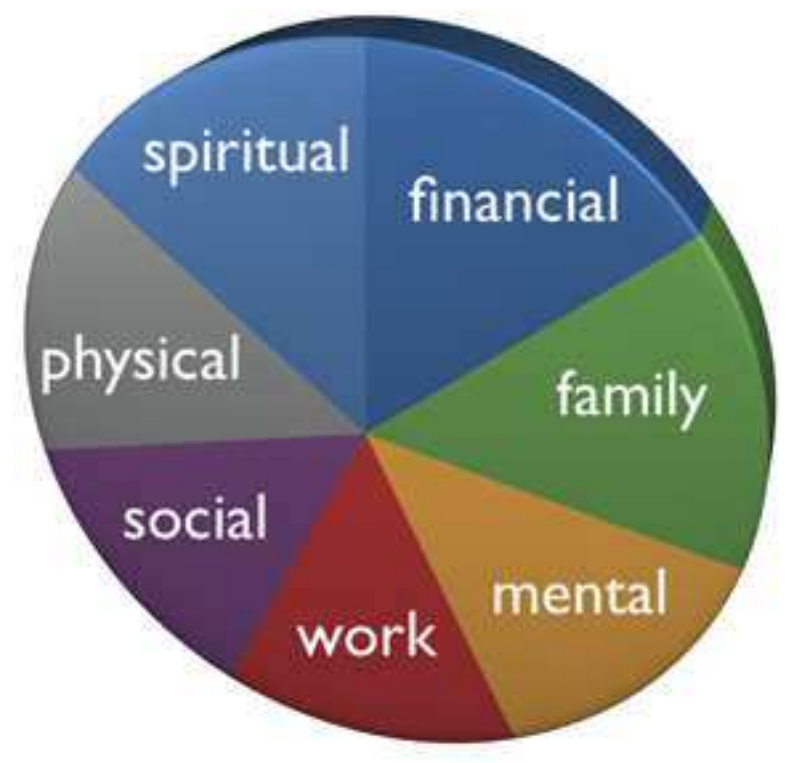

Figure 24. The Balanced Lifestyle Necessary for Recovering from Mental Illness Includes making the Changes for Creating and Living a Balanced Lifestyle.

A balanced lifestyle is a lifestyle in which each element is equal in importance and time devoted stops the progression of mental illness, and allows the FOFR to regain confidence in the conscious, increasing longevity. Researchers estimate that $30 \%$ of longevity is due to heredity and that $70 \%$ is from the overuse of the FOFR.

The balanced lifestyle encompasses many elements that keep people healthy. These elements include developing and maintaining physical health and physical fitness, maintaining a good level of energy and vitality, express your creativity, enjoying emotional and psychological stability with friends and family, entertaining harmonious relationships, feeling and expressing love toward people, family and life. A person's is required to develop and keep effective stress control and management by removing stressors, develop and maintain financial stability and developing a purpose and meaning for life.

A major spiritual perspective gives a sense of belonging and purpose in life. One may experience success in many aspects of their life, but without a sense of belonging and purpose, they will often continue to feel unsatisfied. Deep inside, they feel that something of extreme importance is still missing, but often cannot figure out what it is exactly; they feel a deep painful longing.

To reverse the brain shrinkage caused by mental illnesses, meditation may be successful. The increase in brain size from meditation is based on many scientific studies already completed. Meditation has been shown to increase the size of the brain after several years of devoted practice offering the mentally ill the tool for reversing the reduction in brain size associated with the serious mental illnesses.

\section{Conclusion}

Astronauts in low-earth-orbit experience mental illness, astronauts traveling on deep space missions are expected to experience a variety of severe mental illnesses. These can be predicted, prevented and recovered from by using communication science, predictive science and electrical engineering, physiological and biological data from the astronaut's circadian and ultradian rhythms. Recovery will occur when the working and living environment is changed from an unbalanced, high threat, high stress lifestyle to a balanced, low threat, low stress lifestyle .

The diagnostic biological data from the circadian and ultradian rhythms for predicting and preventing mental illness on deep space missions can be generated and collected during astronaut training, pre-flight readiness, launch and while living and working in space. This data would then be processed with proprietary, data-driven predictive algorithms to illustrate the presence of any premature aging of the brain that precedes mental illness.

The use of the FOFR as the cause of mental illness also explains the cause, treatment for most other diseases when it suppresses the immune system leaving the body vulnerable each time. The toxic chemicals cortisol and adrenaline released by the FOFR were developed to be in the body an extremely short duration. Today's city-based lifestyle force people to remain in threatening situations, as 
determined by the FOFR, forcing cortisol and adrenaline to be present for years at varying levels.

The PHM program identifies any premature aging in biological, diagnostic telemetry by ground personnel who will complete a prognostic analysis and predict mental illness. By using the FOFR as the model for the cause and cure of mental illness, astronaut's mental health can be maintained positive and even improved

PHM uses information obtained from a variety of space mission phases including astronaut readiness, launch vehicle pre-launch readiness, launch and during deep space travel to develop baseline behavior from which premature aging of the brain can be observed allowing mental illness can be predicted so it can be prevented and/or cured.

It is not currently possible to reverse the self-induced brain damage, but scientific studies shows it is possible to reverse the brain shrinkage using serious, long-term meditation programs.

Identifying the FOFR as the cause of mental illness and most other diseases by suppressing the body's immune system on a frequent basis will motivate drugmanufacturing companies to develop drugs to suppress our FOFR, just as drug manufacturers developed drugs to suppress the immune system to treat autoimmune system diseases.

Astronauts living and working in space for long periods suffer major loss of body and brain size and/or functional degradation ion deep space missions. People with serious mental illnesses also suffer from self-induced brain damage including shrinkage of the frontal lobes, which is why their behavior is radically different without medication. The treatment of people with brain damage acquired from living in space and/or mental illness should include neurologists and neuroscientists as well as psychiatrists and psychotherapists.

\section{References}

[1] Maral, Gerard, Bousquet, Michel, "Satellite Communications Systems," Fourth Edition, John Wiley \& Sons, Hoboken, New Jersey, ISBN 0471496545.

[2] Balaji J, Armbruster M, Ryan TA., Journal of Neuroscience, June, 2008， 25;28(26):6742-9. Calcium Control of Endocytic Capacity at a CNS Synapse. (Needs to added)

[3] http://frontierpsychiatrist.co.uk/models-of-mental-illness/

[4] http://cancer.about.com/od/cancercauses/a/Viruses-AndCan-cer.htm

[5] Losik, Len "Using the Brain's Fight-or-Flight Response for Predicting Mental Illness on the Human Space Flight Program," Proceedings from the 2013 IEEE Aerospace Conference, Big Sky Montana.

[6] Circadian Rhythm Laboratory, "Rhythmiccity of the Human Body Vital Signs,) available at the web site at www.Circadian.org/vital.html

[7] Popov, Alex, Médecine spatiale opérationnelle, Operational
Space Medicine Exploration spatiale - Astronautes, sciences de la vie et médecine spatiale Space Exploration Astronauts, Life Sciences and Space Medicine Agence spatiale canadienne, Canadian Space Agency.

[8] Volkov, Sergey, Chief of the Russian Cosmonaut Program. Kazakhstan Baikonur Cosmodrome.

[9] Kanas, N., Sandal, G.M., Boyd, J.E., Gushin, V.I., Manzey, D., North, R., Leon, G.R., Suedfeld, P., Bishop, S., Fiedler, E.R., Inoue, N., et al. 2009. Psychology and culture during long-duration space missions. Acta Astronautica 64:659-677.

[10] Losik, Len, "Upgrading the Space Flight Factory Acceptance Testing for Equipment and Space Vehicle Design, Manufacture, Test and Integration," AIAA Space 2009 Conference proceedings.

[11] Losik, Len, "Predicting Hardware Failures and Estimating Remaining-Usable-Life from Telemetry," SanLen Publishing, Sacramento, CA, 2004, ISBN 978-0-9767491-96.

[12] Failure Analysis, "Data-Driven Predictive Algorithms Users Guide," V2.25, Failure Analysis, Capitola CA.

[13] Packet Telemetry, CCSDS 102.0-B-5, Blue Book, November 2000, CCSDS Secretariat, Program Integration Division (Code MT), National Aeronautics and Space Administration, Washington, DC 20546, USA.

[14] http://science.education.nih.gov/supplements/nih5/Mental/ guide/info-mental-a.htm.

[15] http://www.thevisualmd.com/health_centers/wellness/manag e_your_stress/stress_makes_you_age_faster

[16] Blackburn, Elisabeth, "Stress Makes You Age Faster,", University of California, San Francisco.

[17] http://www.cnsforum.com/imagebank/section/Brain_struc_ anxiety/default.aspx

[18] "Advances In In-Vivo Imaging Of Serotonergic Neurons In Neuropsychiatric Disorders," Swen Hesse, Department of Nuclear Medicine, University Hospital, Henryk Barthel, University of Leipzig, Leipzig, Germany, Johannes Schwarz, Department of Neurology, University Hospital, University of Leipzig, Leipzig, Germany, Osama Sabri, Department of Nuclear Medicine, University Hospital, Ulrich Müller, Departments of Experimental Psychology and Psychiatry, University of Cambridge, Cambridge, United Kingdom, Neuroscience \& Bio-behavioral Reviews, Volume 29, Issue 7, 2005, Page 1119.

[19] Losik, Len "Using the Brain's Fight-or-Flight Response for Predicting Mental Illness on the Human Space Flight Program," Proceedings from the 2013 IEEE Aerospace Conference, Big Sky Montana.

[20] http://english.tebyan.net/newindex.aspx?pid=31159\& BookID $=23649 \&$ Language $=3$

[21] http://science.howstuffworks.com/life/fear2.htm

[22] http://users.ren.com/jkimball.ma.ultranet/BiologyPages/P/ PNS.html

[23] Losik, Len, "Predicting Hardware Failures and Estimating Remaining-Usable-Life from Telemetry," SanLen Publishing, Sacramento, CA, 2004, ISBN 978-0-9767491-96. 
[24] National Institute of Health, National Institute of General Medical Sciences at http://www.nigms.nih.gov/ Education/Factsheet_CircadianRhythms.htm.

[25] http://www.springer.com/life+sciences/book/978-1-4020$8351-8$.
[26] Circadian Rhythm Laboratory, "Rhythmicity of the Human Body Vital Signs,) at www.Circadian.org/vital.html. 